\title{
Spectra of units for periodic ring spectra and group completion of graded $E_{\infty}$ spaces
}

\author{
STEFFEN SAGAVE
}

\begin{abstract}
We construct a new spectrum of units for a commutative symmetric ring spectrum that detects the difference between a periodic ring spectrum and its connective cover. It is augmented over the sphere spectrum. The homotopy cofiber of its augmentation map is a non-connected delooping of the usual spectrum of units whose bottom homotopy group detects periodicity.

Our approach builds on the graded variant of $E_{\infty}$ spaces introduced in joint work with Christian Schlichtkrull. We construct a group completion model structure for graded $E_{\infty}$ spaces and use it to exhibit our spectrum of units functor as a right adjoint on the level of homotopy categories. The resulting group completion functor is an essential tool for studying ring spectra with graded logarithmic structures.
\end{abstract}

$55 \mathrm{P} 43 ; 55 \mathrm{P} 48$

\section{Introduction}

Having a notion of units for ring spectra is useful in connection with algebraic $K$-theory and Thom spectra. Sufficiently commutative ring spectra give rise to spectra of units. This was first made precise by May, Quinn, Ray and Tornehave [16] who defined a spectrum of units associated with an $E_{\infty}$ ring spectrum and showed how it controls its orientations. Ando, Blumberg, Gepner, Hopkins and Rezk [2; 3] generalized this by giving an extensive treatment of units, Thom spectra and orientation theory in the context of structured ring spectra and $\infty$-categories.

In joint work with Schlichtkrull [23], we introduced the graded units $\mathrm{GL}_{1}^{\mathcal{J}} E$ of a commutative symmetric ring spectrum $E$. One key feature of these graded units is that they detect the difference between a periodic ring spectrum and its connective cover, which is essential for applications to logarithmic ring spectra (that are outlined at the end of this introduction). The object $\mathrm{GL}_{1}^{\mathcal{J}} E$ can be viewed as a space-valued lax symmetric monoidal functor on a certain indexing category $\mathcal{J}$. One aim of the present paper is to explain how this approach to graded units leads to spectra of graded units that detect periodicity. To this end, we construct a functor

$$
\mathrm{gl}_{1}^{\mathcal{J}}: \mathcal{C} \operatorname{Sp}^{\Sigma} \rightarrow \Gamma^{\mathrm{op}}-\mathcal{S} / b \mathcal{J}, \quad E \mapsto\left(\mathrm{gl}_{1}^{\mathcal{J}} E \rightarrow b \mathcal{J}\right) .
$$


Here $\mathcal{C S p}^{\Sigma}$ is the category of commutative symmetric ring spectra, $\Gamma^{\mathrm{op}}-\mathcal{S}$ is the category of $\Gamma$-spaces in the sense of Segal, and $b \mathcal{J}$ is a certain explicit $\Gamma$-space coming from a symmetric monoidal category. The spectrum associated with $b \mathcal{J}$ is an $\Omega$-spectrum that is stably equivalent to the sphere spectrum, ie an $\Omega$-spectrum with $0^{\text {th }}$ space $Q S^{0} \simeq \Omega^{\infty} \Sigma^{\infty} S^{0}$. Since $\Gamma$-spaces model connective spectra, the category $\Gamma^{\mathrm{op}}-\mathcal{S} / b \mathcal{J}$ is a convenient model for the category of connective spectra over the sphere spectrum. We explain below why it is reasonable for a spectrum of units to take values in this category, rather than just in connective or non-connective spectra.

Commutative symmetric ring spectra are strictly commutative models for $E_{\infty}$ spectra, and the definition of $\mathrm{gl}_{1}^{\mathcal{J}}$ makes explicit use of their combinatorics. A commutative symmetric ring spectrum $E$ can be defined as a collection of pointed spaces $E_{m}$ together with a basepoint-preserving left action of the symmetric group $\Sigma_{m}$, multiplication maps $E_{m} \wedge E_{n} \rightarrow E_{m+n}$ and unit maps $S^{m} \rightarrow E_{m}$ that satisfy appropriate relations. By definition, the value of the $\Gamma$-space $\operatorname{gl}_{1}^{\mathcal{J}} E$ at the based set $k^{+}=\{0, \ldots, k\}$ is a certain homotopy colimit over a $k$-fold product of subspaces consisting of those path components of $\Omega^{n_{2}} E_{n_{1}}$ that correspond to units in the underlying multiplicative graded monoid of $\pi_{*}(E)$. The augmentation to $b \mathcal{J} \cong \mathrm{gl}_{1}^{\mathcal{J}}(*)$ results from the choice for the indexing category of the homotopy colimits.

\subsection{A non-connected delooping of $\mathrm{gl}_{1} E$}

A first indication for why $\mathrm{gl}_{1}^{\mathcal{J}} E$ is of interest is the following relation to ordinary spectra of units. Let $\operatorname{bgl}_{1}^{*} E$ be the spectrum associated with the homotopy cofiber of the augmentation $\mathrm{gl}_{1}^{\mathcal{J}} E \rightarrow b \mathcal{J}$.

Theorem 1.2 Let $E$ be a positive fibrant commutative symmetric ring spectrum. Then $\operatorname{bgl}_{1}^{*} E$ is a connective spectrum, and the ordinary spectrum of units $\mathrm{gl}_{1} E$ is the connective cover of $\Omega \mathrm{bgl}_{1}^{*} E$.

The bottom homotopy group $\pi_{0}\left(\mathrm{bgl}_{1}^{*} E\right)$ is isomorphic to $\mathbb{Z} / n_{E} \mathbb{Z}$, where $n_{E} \in \mathbb{N}_{0}$ is the periodicity of $E$. By definition, $n_{E}=0$ (and $\mathbb{Z} / n_{E} \mathbb{Z} \cong \mathbb{Z}$ ) if all units of the underlying multiplicative graded monoid of $\pi_{*}(E)$ have degree 0 , and $n_{E}$ is the smallest positive degree of a unit in $\pi_{*}(E)$ otherwise.

The theorem says that $\operatorname{bgl}_{1}^{*} E$ is a not-necessarily-connected delooping of the usual spectrum of units whose bottom homotopy group detects periodicity. If $E$ is for example 2-periodic, then its connective cover $e \rightarrow E$ induces the surjection

$$
\mathbb{Z} \cong \pi_{0}\left(\operatorname{bgl}_{1}^{*} e\right) \rightarrow \pi_{0}\left(\operatorname{bgl}_{1}^{*} E\right) \cong \mathbb{Z} / 2 .
$$


By construction there is a map of spectra $\mathbb{S} \rightarrow \operatorname{bgl}_{1}^{*} E$. The induced map

$$
\mathbb{Z} / 2 \cong \pi_{1}(\mathbb{S}) \rightarrow \pi_{1}\left(\operatorname{bgl}_{1}^{*} E\right) \cong \pi_{0}\left(\mathrm{gl}_{1} E\right) \cong\left(\pi_{0}(E)\right)^{\times}
$$

turns out to be the sign action of the additive group structure on $\pi_{0}(E)$. This relation of the stable Hopf map that generates $\pi_{1}(\mathbb{S})$ and the units does not seem to be visible if one only considers the ordinary $\mathrm{gl}_{1} E$.

A different way of extending the notion of units of $E$ so that periodicity gets detected is to form the Picard space of $E$. By definition, $\operatorname{Pic}(E)$ is the $\infty$-groupoid of invertible elements in the symmetric monoidal $\infty$-category of $E$-modules; see eg Ando, Blumberg and Gepner [1, Section 8]. The bottom homotopy group of $\operatorname{Pic}(E)$ is the Picard group of $E$, and we expect that $\Omega^{\infty}\left(\mathrm{bgl}_{1}^{*} E\right)$ is equivalent to the full $\infty$-subgroupoid of $\operatorname{Pic}(E)$ spanned by the shifts of $E$.

In view of the $E$-algebra Thom spectra associated with maps to $\operatorname{bgl}_{1} E$ or $\operatorname{Pic}(E)$ constructed by Ando, Blumberg, Gepner, Hopkins and Rezk [3] and by Ando, Blumberg and Gepner [1], it is natural to ask if maps to $\operatorname{bgl}_{1}^{*} E$ give rise to graded $E$-algebra Thom spectra. The construction of such a graded Thom spectrum functor will be given in a forthcoming joint project with Schlichtkrull [22]. There we also give an alternative model of the $E_{\infty}$ space $\Omega^{\infty}\left(\operatorname{bgl}_{1}^{*} E\right)$ that can be interpreted as a classifying space for $\mathrm{GL}_{1}^{\mathcal{J}} E$-modules.

\section{$1.3 \mathcal{I}$-spaces and $E_{\infty}$ spaces}

Our main tools for building and studying the functor $\mathrm{gl}_{1}^{\mathcal{J}}$ are methods for the homotopical analysis of diagram spaces developed together with Schlichtkrull in [23]. One important instance for such diagram spaces are $\mathcal{I}$-spaces. These are space-valued functors on the category of finite sets $\boldsymbol{m}=\{1, \ldots, m\}, m \geq 0$, and injective maps. The category of $\mathcal{I}$-spaces $\mathcal{S}^{\mathcal{I}}$ has a symmetric monoidal product $\nabla$ induced by the ordered concatenation of finite sets and the cartesian product of spaces.

The commutative monoids in $\left(\mathcal{S}^{\mathcal{I}}, \bigotimes\right)$ are called commutative $\mathcal{I}$-space monoids and form a category denoted by $\mathcal{C} \mathcal{S}^{\mathcal{I}}$. This category admits a positive $\mathcal{I}$-model structure such that $\mathcal{C} \mathcal{S}^{\mathcal{I}}$ is Quillen equivalent to the category of $E_{\infty}$ spaces. So all $E_{\infty}$ spaces admit a strictly commutative model in $\left(\mathcal{S}^{\mathcal{I}}, \otimes\right)$. The point here is that the extra symmetry of $\mathcal{I}$-spaces and the use of a positive model structure ensure that we do not try to represent the homotopy type of an arbitrary $E_{\infty}$ space by a strictly commutative monoid in spaces (which would be a contradiction). This is related to J Smith's insight that only a positive model structure on symmetric spectra lifts to commutative symmetric ring spectra; see Mandell, May, Schwede and Shipley [14]. 
If $E$ is a commutative symmetric ring spectrum, there is a commutative $\mathcal{I}$-space monoid $\Omega^{\mathcal{I}} E$ representing its multiplicative $E_{\infty}$ space. It is on objects defined by $\left(\Omega^{\mathcal{I}} E\right)(\boldsymbol{m})=\Omega^{m} E_{m}$. The grouplike $E_{\infty}$ space of units of $E$ is represented by a sub commutative $\mathcal{I}$-space monoid $\mathrm{GL}_{1}^{\mathcal{I}} E$ of $\Omega^{\mathcal{I}} E$. It gives rise to a very special $\Gamma$-space $\mathrm{gl}_{1}^{\mathcal{I} E}$ whose associated spectrum models the ordinary spectrum of units; see Schlichtkrull [26] and Lind [12].

The passage from $\mathrm{GL}_{1}^{\mathcal{I}} E$ to $\mathrm{gl}_{1}^{\mathcal{I}} E$ is an implementation of the recognition principle which states that grouplike $E_{\infty}$ spaces have the homotopy types of infinite loop spaces. In [24] we prove in joint work with Schlichtkrull that the category $\mathcal{C S}^{\mathcal{I}}$ admits a group completion model structure $\mathcal{C} \mathcal{S}_{\mathrm{gp}}^{\mathcal{I}}$ whose fibrant objects are positive $\mathcal{I}$-fibrant and grouplike. We further show that $\mathcal{C} \mathcal{S}_{\text {gp }}^{\mathcal{I}}$ is Quillen equivalent to $\Gamma$-spaces with a stable model structure. The resulting equivalence between the homotopy categories of grouplike commutative $\mathcal{I}$-space monoids and connective spectra may be viewed as an incarnation of the recognition principle. We use this Quillen equivalence in [24] to show that the functor of homotopy categories $\operatorname{Ho}\left(\mathcal{C S p}^{\Sigma}\right) \rightarrow \operatorname{Ho}\left(\Gamma^{\mathrm{op}}-\mathcal{S}\right)$ induced by $\mathrm{gl}_{1}^{\mathcal{I}}$ is a right adjoint, reproving a result from [3, Theorem 5.1].

\section{$1.4 \mathcal{J}$-spaces and graded $E_{\infty}$ spaces}

The functor $\mathrm{gl}_{1}^{\mathcal{I}}$ does not detect periodicity because the commutative $\mathcal{I}$-space monoids $\Omega^{\mathcal{I}} E$ and $\mathrm{GL}_{1}^{\mathcal{I}} E$ have no information about the negative-dimensional homotopy groups of $E$. Together with Schlichtkrull we showed in [23] that one can overcome this by using a different indexing category $\mathcal{J}$ instead of $\mathcal{I}$. This $\mathcal{J}$ is defined to be Quillen's localization construction $\Sigma^{-1} \Sigma$ on the category of finite sets and bijections $\Sigma$. It is symmetric monoidal and gives rise to $\mathcal{J}$-spaces and a category of commutative $\mathcal{J}$-space monoids $\mathcal{C S}^{\mathcal{J}}$ just as in the case of $\mathcal{I}$. The classifying space $B \mathcal{J}$ of $\mathcal{J}$ has the homotopy type of $Q S^{0}$. Objects of $\mathcal{J}$ are pairs of finite sets $\left(\boldsymbol{n}_{1}, \boldsymbol{n}_{2}\right)$, and a close relation between $\mathcal{J}$ and the combinatorics of symmetric spectra ensures that there is a functor $\Omega^{\mathcal{J}}: \mathcal{C} \operatorname{Sp}^{\Sigma} \rightarrow \mathcal{C} \mathcal{S}^{\mathcal{J}}$ with $\left(\Omega^{\mathcal{J}} E\right)\left(\boldsymbol{n}_{\mathbf{1}}, \boldsymbol{n}_{2}\right)=\Omega^{n_{2}} E_{n_{1}}$. There is a sub commutative $\mathcal{J}$-space monoid $\mathrm{GL}_{1}^{\mathcal{J}} E$ of $\Omega^{\mathcal{J}} E$ that is used to define the $\Gamma$-space $\mathrm{gl}_{1}^{\mathcal{J}} E$ outlined in (1-1) above.

As for $\mathcal{I}$-spaces, there is a positive $\mathcal{J}$-model structure for $\mathcal{C} \mathcal{S}^{\mathcal{J}}$. With this model structure, $\mathcal{C S}^{\mathcal{J}}$ is related by a chain of Quillen equivalences to the category of $E_{\infty}$ spaces over $B \mathcal{J}$. This result is a reason why we think of commutative $\mathcal{J}$-space monoids as "graded $E_{\infty}$ spaces": while a $\mathbb{Z}$-graded monoid in algebra can be defined as a commutative monoid over the underlying additive monoid $(\mathbb{Z},+)$ of the initial commutative ring $\mathbb{Z}$, a commutative $\mathcal{J}$-space monoid is up to homotopy the same as an $E_{\infty}$ space over $Q S^{0}$, the underlying "additive" infinite loop space of the initial 
commutative ring spectrum $\mathbb{S}$. So $Q S^{0} \simeq B \mathcal{J}$ plays the role of $(\mathbb{Z},+)$. From this point of view, $\Omega^{\mathcal{J}} E$ is the underlying graded multiplicative $E_{\infty}$ space of $E$, and $\mathrm{GL}_{1}^{\mathcal{J}} E$ is the "graded units" of $E$.

\subsection{Group completion of graded commutative spaces}

Combining the last result and the recognition principle mentioned above suggests that grouplike commutative $\mathcal{J}$-space monoids should correspond to connective spectra over the sphere spectrum. One of the main result of the present paper is a proof of this statement on the level of model categories.

Theorem 1.6 The category of commutative $\mathcal{J}$-space monoids admits a group completion model structure in which the fibrant objects are the grouplike positive $\mathcal{J}$-fibrant objects and the fibrant replacement is a group completion.

There is a chain of Quillen equivalences relating this model category to the category of $\Gamma$-spaces over $b \mathcal{J}$ with a stable model structure.

In the theorem, a map of commutative $\mathcal{J}$-space monoids is a group completion if the associated map of $E_{\infty}$ spaces is a group completion in the usual sense. The proof of the theorem is more difficult than (and different to) the proof of the corresponding result about $\mathcal{I}$-spaces in [24]: in the context of $\mathcal{J}$-spaces, the group completion functor $\Omega B$ for simplicial monoids does not lift to $\mathcal{C S}^{\mathcal{J}}$. Moreover, we only get a chain of Quillen equivalences here, rather than a single Quillen equivalence as in the case of $\mathcal{I}$-spaces.

Together with a model category treatment of the passage from $\Omega^{\mathcal{J}} E$ to $\mathrm{GL}_{1}^{\mathcal{J}} E$ that works as in the case of $\mathcal{I}$-spaces, Theorem 1.6 is the key ingredient for:

Theorem 1.7 The functor $\operatorname{Ho}\left(\mathcal{C S p}{ }^{\Sigma}\right) \rightarrow \operatorname{Ho}\left(\Gamma^{\mathrm{op}}-\mathcal{S} / b \mathcal{J}\right)$ induced by $\mathrm{gl}_{1}^{\mathcal{J}}$ is a right adjoint.

This theorem may be viewed as the graded analog of the result about ordinary units mentioned in Section 1.3. Group completions are relevant for this theorem about units because the stably fibrant replacement of a special $\Gamma$-space models the group completion.

\subsection{Applications to ring spectra with logarithmic structures}

A graded pre-log ring spectrum is a commutative symmetric ring spectrum $E$ together with a commutative $\mathcal{J}$-space monoid $M$ and a map $M \rightarrow \Omega^{\mathcal{J}}(E)$ in $\mathcal{C} \mathcal{S}^{\mathcal{J}}$. A variant 
of this notion employing $\mathcal{I}$-spaces instead of $\mathcal{J}$-spaces was introduced by Rognes in [17] in order to use ideas from logarithmic algebraic geometry to understand various phenomena related to topological Hochschild homology and algebraic $K$-theory. As explained in [23, Section 4.30], the $\mathcal{J}$-space version of this definition has the advantage that it is easier to extend topological $K$-theory spectra to (graded) pre-log ring spectra in an interesting way.

The results of the present paper are relevant for the study of pre-log ring spectra. The group completion functor for commutative $\mathcal{J}$-space monoids resulting from Theorem 1.6 is an essential foundation for the author's work on the logarithmic topological André-Quillen homology [21] and the author's joint work with Rognes and Schlichtkrull on logarithmic topological Hochschild homology [19; 20]. For example, the group completion functor is already used in the definition of the logarithmic topological Hochschild homology of graded pre-log ring spectra, and it is a key ingredient for showing that the inclusion of the Adams summand extends to a map of graded pre-log ring spectra which is formally étale through the eyes of logarithmic THH and TAQ. We refer to Rognes' ICM talk [18, Section 7] for a survey of these results.

\subsection{Organization}

We begin with a review of diagram spaces in Section 2 and use this material in Section 3 to define the $\Gamma$-space $\mathrm{gl}_{1}^{\mathcal{J}} E$ appearing in the introduction. Section 4 contains the proof of Theorem 1.2. In Section 5 we give a more detailed formulation of Theorem 1.6 and show how it can be used to prove Theorem 1.7. Sections 6 and 7 are devoted to the construction and identification of group completions needed to prove Theorem 1.6.

\subsection{Conventions}

In this paper, space means unpointed simplicial set. Most proofs would work equally well for compactly generated weak Hausdorff spaces, although some arguments in connection with localizations of model categories differ. When using the symbols $\rightleftarrows$ and $\leftrightarrows$ for (possibly unlabeled) adjoint pairs of functors, we always denote the left adjoint on the top. We assume some familiarity with model categories and mostly use Hirschhorn's book [10] as a reference.

Acknowledgments The author would like to thank Christian Schlichtkrull and Stefan Schwede for helpful conversations related to this project, and John Rognes for comments on an earlier version of the manuscript. Moreover, the author would like to thank the unnamed referee for helpful comments on an earlier version of this manuscript. 


\section{Structured diagram spaces and units}

In this section we review some terminology about diagram spaces from our joint work with Schlichtkrull [23]. For a commutative symmetric ring spectrum $E$, we define its units $\mathrm{GL}_{1}^{\mathcal{I}} E$, its graded units $\mathrm{GL}_{1}^{\mathcal{J}} E$, and the diagram categories in which these objects are defined.

\subsection{Diagram spaces}

We begin with some general definitions that will be used frequently throughout the paper.

Definition 2.2 Let $\mathcal{K}$ be a small category. A $\mathcal{K}$-space is a functor $\mathcal{K} \rightarrow \mathcal{S}$ to the category of (unpointed) simplicial sets. We write $\mathcal{S}^{\mathcal{K}}$ for the category of $\mathcal{K}$-spaces.

A map of $\mathcal{K}$-spaces $f: X \rightarrow Y$ is a level equivalence if $f(\boldsymbol{k}): X(\boldsymbol{k}) \rightarrow Y(\boldsymbol{k})$ is a weak equivalence for every object $\boldsymbol{k}$ of $\mathcal{K}$. Level equivalences are often too rigid, and the following weaker notion of equivalence is useful for many purposes.

Definition 2.3 A map $f: X \rightarrow Y$ of $\mathcal{K}$-spaces is a $\mathcal{K}$-equivalence if it induces a weak equivalence $f_{h \mathcal{K}}: X_{h \mathcal{K}} \rightarrow Y_{h \mathcal{K}}$ of homotopy colimits. Here

$$
X_{h \mathcal{K}}=\operatorname{hocolim}_{\mathcal{K}} X=\operatorname{diag}\left([i] \mapsto \coprod_{\boldsymbol{k}_{\mathbf{0}} \leftarrow \cdots \leftarrow \boldsymbol{k}_{\boldsymbol{i}}} X\left(\boldsymbol{k}_{\boldsymbol{i}}\right)\right)
$$

is the usual Bousfield-Kan homotopy colimit of a $\mathcal{K}$-space $X$.

Now let $\mathcal{K}$ be a small symmetric monoidal category with product $\sqcup$ and monoidal unit $\mathbf{0}$. Then the category of $\mathcal{K}$-spaces has a symmetric monoidal product $\boldsymbol{\nabla}$ induced by the symmetric monoidal structure of $\mathcal{K}$ and the cartesian product of spaces: for $\mathcal{K}$-spaces $X$ and $Y$, we define $X \otimes Y$ as the left Kan extension of the object-wise cartesian product along $\sqcup: \mathcal{K} \times \mathcal{K} \rightarrow \mathcal{K}$. The unit for $\nabla$ is the $\mathcal{K}$-space $\mathbf{1}_{\mathcal{K}}=\mathcal{K}(\mathbf{0},-)$.

Definition 2.4 A commutative monoid in $\left(\mathcal{S}^{\mathcal{K}}, \otimes, \mathbf{1}_{\mathcal{K}}\right)$ is called a commutative $\mathcal{K}$ space monoid, and $\mathcal{C} \mathcal{S}^{\mathcal{K}}$ denotes the category of commutative $\mathcal{K}$-space monoids.

Unraveling the definition of a left Kan extension, a commutative $\mathcal{K}$-space monoid is a $\mathcal{K}$-space $A$ together with multiplication maps $A(\boldsymbol{k}) \times A(\boldsymbol{l}) \rightarrow A(\boldsymbol{k} \sqcup \boldsymbol{l})$ and a unit map $* \rightarrow A(\mathbf{0})$ which are associative, unital and commutative.

For a commutative $\mathcal{K}$-space monoid $A$, the homotopy colimit $A_{h \mathcal{K}}$ inherits the structure of a simplicial monoid with product

$$
A_{h \mathcal{K}} \times A_{h \mathcal{K}} \cong \operatorname{hocolim}_{(\boldsymbol{k}, \boldsymbol{l}) \in \mathcal{K} \times \mathcal{K}} A(\boldsymbol{k}) \times A(\boldsymbol{l}) \rightarrow \operatorname{hocolim}_{(\boldsymbol{k}, \boldsymbol{l}) \in \mathcal{K} \times \mathcal{K}} A(\boldsymbol{k} \sqcup \boldsymbol{l}) \rightarrow A_{h \mathcal{K}} .
$$


Here the first map is induced by the multiplication of $A$ and the second map induced by the monoidal structure of $\mathcal{K}$. The monoid $A_{h \mathcal{K}}$ is usually not commutative since the symmetry isomorphism of $\mathcal{K}$ may differ from the identity. However, commutativity of $A$ implies that the monoid of path components $\pi_{0}\left(A_{h \mathcal{K}}\right)$ of $A_{h \mathcal{K}}$ is commutative.

Definition 2.5 Let $A$ be a commutative $\mathcal{K}$-space monoid.

(i) $A$ is grouplike if the commutative monoid $\pi_{0}\left(A_{h \mathcal{K}}\right)$ is a group.

(ii) The units $A^{\times}$of $A$ is the grouplike sub commutative $\mathcal{K}$-space monoid of $A$ with $A^{\times}(\boldsymbol{k})$ consisting of those components of $A(\boldsymbol{k})$ that map to a unit in the commutative monoid $\pi_{0}\left(A_{h \mathcal{K}}\right)$.

One can check that $A^{\times}$is well defined and that $A^{\times} \rightarrow A$ realizes the inclusion $\left(\pi_{0}\left(A_{h \mathcal{K}}\right)\right)^{\times} \rightarrow \pi_{0}\left(A_{h \mathcal{K}}\right)$.

\section{$2.6 \mathcal{I}$-spaces and units}

The category of finite sets and injections $\mathcal{I}$ is one indexing category for diagram spaces which is of importance for us.

Definition 2.7 Let $\mathcal{I}$ be the category with objects the sets $\boldsymbol{m}=\{1, \ldots, m\}$ for $m \geq 0$ and morphisms the injective maps. The ordered concatenation $\sqcup$ of ordered sets makes $\mathcal{I}$ a permutative category, ie a symmetric monoidal category with strict unit and associativity. The monoidal unit is the empty set $\mathbf{0}$ and the symmetry isomorphism the shuffle $\chi_{m, n}: \boldsymbol{m} \sqcup \boldsymbol{n} \rightarrow \boldsymbol{n} \sqcup \boldsymbol{m}$ moving the first $m$ elements past the last $n$ elements.

We obtain a category $\mathcal{C} \mathcal{S}^{\mathcal{I}}$ of commutative $\mathcal{I}$-space monoids. This category is useful because it admits a positive $\mathcal{I}$-model structure such that $\mathcal{C S}^{\mathcal{I}}$ is Quillen equivalent to the category of $E_{\infty}$ spaces over an $E_{\infty}$ operad [23, Theorem 1.2]. The weak equivalences in the positive $\mathcal{I}$-model structure are the $\mathcal{I}$-equivalences of Definition 2.3. So all homotopy types of $E_{\infty}$ spaces are represented by commutative $\mathcal{I}$-space monoids. In many examples one can write down explicit models for such commutative $\mathcal{I}$-space monoids. We now review from [26;23] how this is done for the units of a commutative symmetric ring spectrum.

A commutative symmetric ring spectrum $E$ may be described by a sequence of pointed spaces $E_{n}$ for $n \geq 0$ with basepoint-preserving left $\Sigma_{n}$-actions on $E_{n}$, multiplication maps $E_{m} \wedge E_{n} \rightarrow E_{m+n}$ and unit maps $S^{n} \rightarrow E_{n}$ satisfying appropriate associativity, commutativity and unitality conditions; see Schwede [28, Section I.1]. In this description, the iterated structure map $\sigma: E_{m} \wedge S^{n} \rightarrow E_{m+n}$ of the underlying symmetric 
spectrum of $E$ is the composite $E_{m} \wedge S^{n} \rightarrow E_{m} \wedge E_{n} \rightarrow E_{m+n}$ of the unit and the multiplication. We write $\mathcal{C} \operatorname{Sp}^{\Sigma}$ for the category of commutative symmetric ring spectra. The commutative $\mathcal{I}$-space monoid $\Omega^{\mathcal{I}} E$ modeling the underlying multiplicative $E_{\infty}$ space of $E$ is defined as follows. Its value at an object $\boldsymbol{m}$ in $\mathcal{I}$ is the space $\Omega^{m}\left(E_{m}\right)$. To define the action of a morphism $\alpha: \boldsymbol{m} \rightarrow \boldsymbol{n}$ in $\mathcal{I}$, choose an extension $\pi: \boldsymbol{n} \rightarrow \boldsymbol{n}$ of $\alpha$ to a bijection and define $\alpha_{*}(f)$ for a zero simplex $f: S^{m} \rightarrow E_{m}$ in $\Omega^{m}\left(E_{m}\right)$ to be the composite

$$
S^{n} \stackrel{\pi_{*}^{-1}}{\longrightarrow} S^{n} \cong S^{m} \wedge S^{n-m} \stackrel{f \wedge \mathrm{id}}{\longrightarrow} E_{m} \wedge S^{n-m} \stackrel{\sigma}{\rightarrow} E_{n} \stackrel{\pi_{*}}{\longrightarrow} E_{n} .
$$

This does not depend on the choice of $\pi$ and extends to a morphism of mapping spaces $\Omega^{m}\left(E_{m}\right) \rightarrow \Omega^{n}\left(E_{n}\right)$ that makes $\Omega^{\mathcal{I}} E$ an $\mathcal{I}$-space. The multiplication of $E$ defines maps $\Omega^{m}\left(E_{m}\right) \times \Omega^{n}\left(E_{n}\right) \rightarrow \Omega^{m+n}\left(E_{m+n}\right)$ by sending $f: S^{m} \rightarrow E_{m}$ and $g: S^{n} \rightarrow E_{n}$ to

$$
S^{m+n} \cong S^{m} \wedge S^{n} \stackrel{f \wedge g}{\longrightarrow} E_{m} \wedge E_{n} \rightarrow E_{m+n},
$$

and $\Omega^{\mathcal{I}} E$ becomes a commutative $\mathcal{I}$-space monoid with this structure.

One can only expect $\Omega^{\mathcal{I}} E$ to be homotopically well behaved for sufficiently fibrant $E$. (Already the mapping spaces involved are not homotopy invariant for general simplicial sets.) We will therefore only apply $\Omega^{\mathcal{I}}$ to fibrant objects in the positive model structure on $\mathcal{C} \operatorname{Sp}^{\Sigma}$ introduced by Mandell, May, Schwede and Shipley [14]. By definition, $E$ is positive fibrant if for $m \geq 1$ the $E_{m}$ are fibrant as simplicial sets and the adjoint structure maps $E_{m} \rightarrow \Omega\left(E_{m+1}\right)$ are weak equivalences. In fact, $\Omega^{\mathcal{I}}$ is a right Quillen functor with respect to the positive model structure on $\mathcal{C S p}^{\Sigma}$ and the positive $\mathcal{I}$-model structure on $\mathcal{C S}^{\mathcal{I}}$ [23, Proposition 3.19(ii)].

Definition 2.8 Let $E$ be a positive fibrant commutative symmetric ring spectrum. The units $\mathrm{GL}_{1}^{\mathcal{I}} E$ of $E$ is the commutative $\mathcal{I}$-space monoid $\left(\Omega^{\mathcal{I}} E\right)^{\times}$.

For such $E$, the fact that the classifying space $B \mathcal{I}$ is contractible can be used to show that the commutative monoid $\pi_{0}\left(\left(\Omega^{\mathcal{I}} E\right)_{h \mathcal{I}}\right)$ is isomorphic to the underlying multiplicative monoid of the $0^{\text {th }}$ stable homotopy group $\pi_{0}(E)$ of $E$. The sub commutative $\mathcal{I}$-space monoid $\mathrm{GL}_{1}^{\mathcal{I}} E$ is characterized by $\pi_{0}\left(\mathrm{GL}_{1}^{\mathcal{I}} E\right)_{h \mathcal{I}} \cong\left(\pi_{0}(E)\right)^{\times}$. It provides the desired strictly commutative model for the units of $E$.

\section{$2.9 \mathcal{J}$-spaces and graded units}

Our second example of an indexing category for diagram spaces will lead to the graded units outlined in the introduction. 
Definition 2.10 [23, Definition 4.2] Let $\mathcal{J}$ be the category whose objects are pairs $\left(\boldsymbol{m}_{1}, \boldsymbol{m}_{\mathbf{2}}\right)$ of objects in $\mathcal{I}$. There are no morphisms $\left(\boldsymbol{m}_{\mathbf{1}}, \boldsymbol{m}_{\mathbf{2}}\right) \rightarrow\left(\boldsymbol{n}_{\mathbf{1}}, \boldsymbol{n}_{\mathbf{2}}\right)$ unless $m_{2}-m_{1}=n_{2}-n_{1}$, and in this case a morphism $\left(\boldsymbol{m}_{\mathbf{1}}, \boldsymbol{m}_{\mathbf{2}}\right) \rightarrow\left(\boldsymbol{n}_{\mathbf{1}}, \boldsymbol{n}_{\mathbf{2}}\right)$ is a triple $\left(\beta_{1}, \beta_{2}, \sigma\right)$ with the $\beta_{i}: \boldsymbol{m}_{\boldsymbol{i}} \rightarrow \boldsymbol{n}_{\boldsymbol{i}}$ morphisms in $\mathcal{I}$ and $\sigma: \boldsymbol{n}_{\mathbf{1}} \backslash \beta_{1}\left(\boldsymbol{m}_{\mathbf{1}}\right) \rightarrow \boldsymbol{n}_{\mathbf{2}} \backslash \beta_{2}\left(\boldsymbol{m}_{\mathbf{2}}\right)$ a bijection identifying the complements of $\beta_{1}$ and $\beta_{2}$.

This $\mathcal{J}$ is indeed a category with the composite

$$
\left(\boldsymbol{l}_{\mathbf{1}}, \boldsymbol{l}_{\mathbf{2}}\right) \stackrel{\left(\alpha_{1}, \alpha_{2}, \rho\right)}{\longrightarrow}\left(\boldsymbol{m}_{\mathbf{1}}, \boldsymbol{m}_{\mathbf{2}}\right) \stackrel{\left(\beta_{1}, \beta_{2}, \sigma\right)}{\longrightarrow}\left(\boldsymbol{n}_{\mathbf{1}}, \boldsymbol{n}_{\mathbf{2}}\right)
$$

defined to be the composite $\beta_{i} \alpha_{i}$ in the first two components and the bijection $\sigma \cup \beta_{2} \rho \beta_{1}^{-1}$ in the last component. Concatenation in both entries makes $\mathcal{J}$ a permutative category.

As before let $E$ be a commutative symmetric ring spectrum. The category $\mathcal{J}$ is made up so that there is a commutative $\mathcal{J}$-space monoid $\Omega^{\mathcal{J}} E$ whose value at an object $\left(\boldsymbol{m}_{1}, \boldsymbol{m}_{2}\right)$ is the space $\Omega^{m_{2}}\left(E_{m_{1}}\right)$. To describe the action of a morphism $\left(\beta_{1}, \beta_{2}, \sigma\right):\left(\boldsymbol{m}_{\mathbf{1}}, \boldsymbol{m}_{\mathbf{2}}\right) \rightarrow\left(\boldsymbol{n}_{\mathbf{1}}, \boldsymbol{n}_{2}\right)$ in $\mathcal{J}$, we choose two bijections $\pi_{i}: \boldsymbol{n}_{\boldsymbol{i}} \rightarrow \boldsymbol{n}_{\boldsymbol{i}}$ extending the $\beta_{i}$ such that $\sigma\left(\pi_{1}\left(m_{1}+j\right)\right)=\pi_{2}\left(m_{2}+j\right)$ for $1 \leq j \leq n_{1}-m_{1}=n_{2}-m_{2}$. On a zero simplex $f: S^{m_{2}} \rightarrow E_{m_{1}}$ of $\Omega^{m_{2}}\left(E_{m_{1}}\right)$, the induced map $\left(\beta_{1}, \beta_{2}, \sigma\right)_{*}(f)$ is defined as the composite

$$
S^{n_{2}} \stackrel{\left(\pi_{2}^{-1}\right)_{*}}{\longrightarrow} S^{n_{2}} \cong S^{m_{2}} \wedge S^{n_{2}-m_{2}} \stackrel{f \wedge \text { id }}{\longrightarrow} E_{m_{1}} \wedge S^{n_{1}-m_{1}} \stackrel{\sigma}{\rightarrow} E_{n_{1}} \stackrel{\left(\pi_{1}\right)_{*}}{\longrightarrow} E_{n_{1}} .
$$

As in the case of $\mathcal{I}$-spaces, this does not depend on the choice of the $\pi_{i}$ and extends to a morphism of mapping spaces $\Omega^{m_{2}}\left(E_{m_{1}}\right) \rightarrow \Omega^{n_{2}}\left(E_{n_{1}}\right)$ that makes $\Omega^{\mathcal{J}} E$ a $\mathcal{J}$-space. The monoid structure on $\Omega^{\mathcal{J}} E$ is defined similarly as for $\Omega^{\mathcal{I}} E$.

Again, $\Omega^{\mathcal{J}}$ is not homotopy invariant on the whole category $\mathcal{C} \operatorname{Sp}^{\Sigma}$. Since it is a right Quillen functor with respect to the positive stable model structure on $\mathcal{C} \operatorname{Sp}^{\Sigma}$ and the positive $\mathcal{J}$-model structure on $\mathcal{C} \mathcal{S}^{\mathcal{J}}$ to be reviewed in Section 5, we only apply $\Omega^{\mathcal{J}}$ to positive fibrant commutative symmetric ring spectra.

Definition 2.11 Let $E$ be a commutative symmetric ring spectrum. The graded units $\mathrm{GL}_{1}^{\mathcal{J}} E$ of $E$ is the commutative $\mathcal{J}$-space monoid $\left(\Omega^{\mathcal{J}} E\right)^{\times}$.

The graded units $\mathrm{GL}_{1}^{\mathcal{J}} E$ now captures the units in all degrees of the graded commutative ring $\pi_{*}(E)$ : in [23, Section 4.14] we prove that one can associate a graded signed monoid $\pi_{0, *}(A)$ to a commutative $\mathcal{J}$-space monoid $A$. Here a graded signed monoid is a $\mathbb{Z}$-graded monoid $M_{*}$ together with $\{ \pm 1\}$-actions on each $M_{S}$ such that $a b=(-1)^{s t} b a$ holds for $a \in M_{s}$ and $b \in M_{t}$. For $\pi_{0, *}\left(\Omega^{\mathcal{J}} E\right)$, the $\{ \pm 1\}$-action is the 
additive sign action of the graded ring $\pi_{*}(E)$. The inclusion $\mathrm{GL}_{1}^{\mathcal{J}} E \rightarrow \Omega^{\mathcal{J}} E$ realizes the inclusion $\pi_{*}(E)^{\times} \rightarrow \pi_{*}(E)$ under $\pi_{0, *}(-)$.

There is an important difference to the situation of $\mathcal{I}$-spaces. The graded signed monoid $\pi_{0}(A)$ of a commutative $\mathcal{J}$-space monoid $A$ is not simply given by $\pi_{0}\left(A_{h \mathcal{J}}\right)$. As we will see in Proposition 4.6, the reason behind this is that the classifying space $B \mathcal{J}$ of $\mathcal{J}$ is not contractible, but rather homotopy equivalent to $Q S^{0}$. However, these two monoids are related: $\pi_{0}\left(A_{h \mathcal{J}}\right)$ is isomorphic to the quotient of the underlying ungraded monoid of $\pi_{0}(A)$ by the $\{ \pm 1\}$-action [23, Corollary 4.17].

A commutative $\mathcal{J}$-space monoid $A$ has an underlying commutative $\mathcal{I}$-space monoid: there is a strong symmetric monoidal diagonal functor $\Delta: \mathcal{I} \rightarrow \mathcal{J}$ with $\Delta(\boldsymbol{m})=(\boldsymbol{m}, \boldsymbol{m})$ and $\Delta(\alpha: \boldsymbol{m} \rightarrow \boldsymbol{n})=\left(\alpha, \alpha, \operatorname{id}_{\boldsymbol{n} \backslash \alpha(\boldsymbol{m})}\right)$, and it is easy to see that $\Delta^{*}(A)=A \circ \Delta$ is a commutative $\mathcal{I}$-space monoid. This recovers $\mathrm{GL}_{1}^{\mathcal{I}} E$ from $\mathrm{GL}_{1}^{\mathcal{J}} E$ :

Lemma 2.12 There is a natural isomorphism $\Delta^{*}\left(\mathrm{GL}_{1}^{\mathcal{J}} E\right) \cong \mathrm{GL}_{1}^{\mathcal{I}} E$.

Proof The isomorphism $\Delta^{*}\left(\Omega^{\mathcal{J}} E\right) \cong \Omega^{\mathcal{I}} E$ is evident, and a 0 -simplex in $\Omega^{m}\left(E_{m}\right)$ maps to an invertible path component of $\left(\Omega^{\mathcal{J}} E\right)_{h \mathcal{J}}$ if and only if it maps to an invertible path component of $\left(\Omega^{\mathcal{I}} E\right)_{h \mathcal{I}}$.

\section{$3 \quad \Gamma$-spaces associated with structured diagram spaces}

The aim of this section is to build $\Gamma$-spaces whose associated spectra provide deloopings of the simplicial monoids $\left(\mathrm{GL}_{1}^{\mathcal{I}} E\right)_{h \mathcal{I}}$ and $\left(\mathrm{GL}_{1}^{\mathcal{J}} E\right)_{h \mathcal{J}}$. The construction will involve deloopings of the respective indexing categories that we construct first. Since it covers the relevant examples and simplifies the bookkeeping, we restrict ourselves to permutative indexing categories.

\section{$3.1 \Gamma$-spaces}

We begin with recalling some basic terminology about $\Gamma$-spaces from Segal [29] and Bousfield and Friedlander [7]. Let $\Gamma^{\mathrm{op}}$ be the category of finite based sets. We write $k^{+}$ for the object of $\Gamma^{\mathrm{op}}$ given by the set $\{0, \ldots, k\}$ with basepoint 0 . A $\Gamma$-space is a covariant functor $X: \Gamma^{\mathrm{op}} \rightarrow \mathcal{S}$ such that $X\left(0^{+}\right)=*$. The $\Gamma$-space $X$ is called special if for each pair of finite based sets $(S, T)$ the natural map $X(S \vee T) \rightarrow X(S) \times X(T)$ induced by the projections $S \vee T \rightarrow S$ and $S \vee T \rightarrow T$ is a weak equivalence. For a special $\Gamma$-space $X$ the set of path components $\pi_{0}\left(X\left(1^{+}\right)\right)$has the structure of a commutative monoid, and $X$ is called very special if this monoid is a group. A $\Gamma$-space $X$ has an associated (sequential) spectrum $X(\mathbb{S})$ with $X(\mathbb{S})_{0}=X\left(1^{+}\right)$. If $X$ is very special, the level fibrant replacement of $X(\mathbb{S})$ is an $\Omega$-spectrum and therefore exhibits $X(\mathbb{S})_{0}=X\left(1^{+}\right)$as an infinite loop space. 


\subsection{Delooping permutative categories}

We now explain how to deloop the classifying space $B \mathcal{K}$ of a small permutative category. The idea is to build a special $\Gamma$-space from $\mathcal{K}$ whose associated spectrum is a delooping of the simplicial monoid $B \mathcal{K}$ if $\pi_{0}(B \mathcal{K})$ is a group. The naive choice would be to try to define a $\Gamma$-space by $S \mapsto B\left(\mathcal{K}^{\times \bar{S}}\right)$, where $\mathcal{K}^{\times \bar{S}}$ denotes a product of copies of $\mathcal{K}$ indexed by the set $\bar{S}=S \backslash 0^{+}$. However, this cannot be made functorial in $\Gamma^{\mathrm{op}}$ : the objects of $\Gamma^{\mathrm{op}}$ are unordered sets, while a non-trivial symmetry isomorphism of $\mathcal{K}$ makes it necessary to specify an ordering of the factors before using the monoidal product to define the structure maps of the $\Gamma$-space. The following construction by Shimada and Shimakawa (see [30] or [8, Section 2.3.1]) avoids this problem.

Definition 3.3 Let $(\mathcal{K}, \sqcup, 0)$ be a permutative category. For an object $S$ in $\Gamma^{\text {op }}$, let $H \mathcal{K}(S)$ be the category whose objects are maps $s: \mathcal{P}(\bar{S}) \rightarrow \mathrm{Ob}(\mathcal{K})$ from the power set of $\bar{S}=S \backslash 0^{+}$to the set of objects of $\mathcal{K}$ together with isomorphisms $\sigma_{U, V}: s_{U} \sqcup s_{V} \rightarrow s_{U \cup V}$ for every pair of disjoint subsets $U, V \subseteq \bar{S}$ such that the following conditions hold:

(i) $\boldsymbol{s}_{\varnothing}=\mathbf{0}$.

(ii) $\sigma_{\varnothing, V}: \mathbf{0} \sqcup \boldsymbol{s}_{V} \rightarrow \boldsymbol{s}_{V}$ and $\sigma_{U, \varnothing}: \boldsymbol{s}_{U} \sqcup \mathbf{0} \rightarrow \boldsymbol{s}_{U}$ are the identity.

(iii) $\sigma_{U \cup V, W} \circ\left(\sigma_{U, V} \sqcup s_{W}\right)=\sigma_{U, V \cup W} \circ\left(s_{U} \sqcup \sigma_{V, W}\right)$ as maps $\boldsymbol{s}_{U} \sqcup \boldsymbol{s}_{V} \sqcup \boldsymbol{s}_{W} \rightarrow$ $s_{U \cup V \cup W}$.

(iv) $\sigma_{V, U} \circ \chi_{\boldsymbol{s}_{U}, \boldsymbol{s}_{V}}=\sigma_{U, V}$ as maps $\boldsymbol{s}_{U} \sqcup \boldsymbol{s}_{V} \rightarrow \boldsymbol{s}_{U \cup V}$.

Morphisms $f:(\boldsymbol{s}, \sigma) \rightarrow(\boldsymbol{t}, \tau)$ are families of morphisms $f_{U}: \boldsymbol{s}_{U} \rightarrow \boldsymbol{t}_{U}$ such that $f_{\varnothing}=\operatorname{id}_{\mathbf{0}}$ and $\tau_{U, V} \circ\left(f_{U} \sqcup f_{V}\right)=f_{U \cup V} \circ \sigma_{U, V}$ hold.

It is easy to see that $H \mathcal{K}(S)$ is again a permutative category. Its monoidal unit $\mathbf{0}_{S}$ is the map $\mathcal{P}(\bar{S}) \rightarrow \operatorname{Ob}(\mathcal{K})$ with constant value $\mathbf{0}$.

For a map $\alpha: S \rightarrow T$ in $\Gamma^{\text {op }}$ and an object $(s, \sigma)$ in $H \mathcal{K}(S)$, we set

$$
\alpha_{*}(s)=\left(\mathcal{P}(\bar{T}) \stackrel{\alpha^{-1}}{\longrightarrow} \mathcal{P}(\bar{S}) \stackrel{s}{\rightarrow} \mathrm{Ob}(\mathcal{K})\right)
$$

and $\alpha_{*}(\sigma)_{U, V}=\sigma_{\alpha^{-1}(U), \alpha^{-1}(V)}$ in order to define an object $\alpha_{*}(s, \sigma)$ of $H \mathcal{K}(T)$.

Lemma 3.4 This defines a covariant functor $H \mathcal{K}$ from $\Gamma^{\mathrm{op}}$ to the category of categories. Evaluation of an object at the one-element subsets of $S$ induces an equivalence of categories $H \mathcal{K}(S) \rightarrow \mathcal{K}^{\times} \bar{S}$. 
Proof The functoriality is immediate. The choice of an ordering of $\bar{S}$ induces an inverse to the functor $H \mathcal{K}(S) \rightarrow \mathcal{K} \times \bar{S}$.

Definition 3.5 We write $b \mathcal{K}$ for the $\Gamma$-space obtained by composing $H \mathcal{K}$ with the classifying space functor.

The $\Gamma$-space $b \mathcal{K}$ is also known as the algebraic $K$-theory of $\mathcal{K}$; see Dundas, Goodwillie and McCarthy [8, Section 2.3.1].

Corollary 3.6 The $\Gamma$-space $b \mathcal{K}$ is special. If the monoid of path components $\pi_{0}(B \mathcal{K})$ of $\mathcal{K}$ is a group, then $b \mathcal{K}$ is very special.

Example 3.7 The very special $\Gamma$-space $b \mathcal{I}$ associated with the category $\mathcal{I}$ is not very interesting: it is levelwise contractible because $\mathcal{I}$ has an initial object.

Example 3.8 It is shown in [23, Proposition 4.4] that the category $\mathcal{J}$ is equivalent to Quillen's localization construction $\Sigma^{-1} \Sigma$ on the category of finite sets and bijections $\Sigma$. Hence its classifying space $B \mathcal{J}$ is equivalent to $Q S^{0}$ by the Barratt-Priddy-Quillen theorem. Segal's version of the Barratt-Priddy-Quillen theorem [29] implies that the special $\Gamma$-space $b \Sigma$ is stably equivalent to the $\Gamma$-space $\Gamma^{\mathrm{op}}\left(1^{+},-\right)$representing the sphere spectrum. Since the functor $\Sigma \rightarrow \Sigma^{-1} \Sigma=\mathcal{J}$ induces a group completion $B \Sigma \rightarrow B \mathcal{J}$, the map $b \Sigma \rightarrow b \mathcal{J}$ is a stable equivalence of $\Gamma$-spaces. So the very special $\Gamma$-space $b \mathcal{J}$ represents the sphere spectrum. The associated spectrum $b \mathcal{J}(\mathbb{S})$ is thus (up to level fibrant replacement) an $\Omega$-spectrum representing the homotopy type of the sphere spectrum. As opposed to other $\Omega$-spectra modeling the sphere spectrum, its definition does not involve taking a loop space or forming a telescope.

\section{9 $\Gamma$-spaces from commutative $\mathcal{K}$-space monoids}

In [26], Schlichtkrull constructs $\Gamma$-spaces from commutative $\mathcal{I}$-space monoids. We give a description of his approach that applies to both commutative $\mathcal{I}$ - and $\mathcal{J}$-space monoids.

Let $\mathcal{K}$ be a small permutative category, let $A$ be a commutative $\mathcal{K}$-space monoid, and let $H \mathcal{K}$ be the $\Gamma$-category associated with $\mathcal{K}$. For every object $S$ in $\Gamma^{\text {op }}$, we define a $H \mathcal{K}(S)$-space $A_{S}$ by

$$
(s, \sigma) \mapsto \prod_{i \in \bar{S}} A\left(s_{i}\right)
$$

Here we write $s_{i}$ for $s(\{i\})$. 
We saw that a map $\alpha: S \rightarrow T$ in $\Gamma^{\text {op }}$ induces a functor $\alpha_{*}: H \mathcal{K}(S) \rightarrow H \mathcal{K}(T)$. There is an induced functor $\alpha^{*}: \mathcal{S}^{H \mathcal{K}(T)} \rightarrow \mathcal{S}^{H \mathcal{K}(S)}$ sending $A_{T}$ to $\alpha^{*}\left(A_{T}\right)=A_{T} \circ \alpha_{*}$. The value of $\alpha^{*}\left(A_{T}\right)$ at an object $(\boldsymbol{s}, \sigma)$ of $H \mathcal{K}(S)$ is

$$
\prod_{j \in \bar{T}} A\left(s\left(\alpha^{-1}(j)\right)\right)
$$

The structure maps of the commutative $\mathcal{K}$-space monoid $A$ induce a map

$$
\tilde{\alpha}: A_{S} \rightarrow \alpha^{*}\left(A_{T}\right)
$$

of $H \mathcal{K}(S)$-spaces: for every element $j \in \bar{T}$, we choose an ordering of the set $V=$ $\alpha^{-1}(j)$ and define the map to be the product over all $j \in \bar{T}$ of the maps

$$
\prod_{i \in V} A\left(s_{i}\right) \rightarrow A\left(\bigsqcup_{i \in V} s_{i}\right) \rightarrow A\left(s_{V}\right)
$$

Here the iterated monoidal product in $\mathcal{K}$ in the middle term is formed using the chosen ordering of $V$, the first map comes from the product of $A$, and the last map is induced by the isomorphisms $\sigma$ that belong to the object of $H \mathcal{K}(S)$ in question. The commutativity of $A$ ensures that the map (3-2) does not depend on the choice of the ordering. If $\alpha^{-1}(j)$ is empty, the map (3-2) is the unit $* \rightarrow A(\mathbf{0})$ of $A$.

Lemma 3.10 For maps $\alpha: S \rightarrow T$ and $\beta: T \rightarrow U$ in $\Gamma^{\mathrm{op}}$, the composite $\left(\alpha^{*}(\tilde{\beta})\right) \tilde{\alpha}$ and $\widetilde{\beta \alpha}$ coincide as maps $A_{S} \rightarrow \alpha^{*}\left(\beta^{*} A_{U}\right)=(\beta \alpha)^{*} A_{U}$.

Definition 3.11 The $\Gamma$-space $\gamma(A)$ associated with a commutative $\mathcal{K}$-space monoid $A$ is defined by $\gamma(A)(S)=$ hocolim $_{H \mathcal{K}(S)} A_{S}$ on the objects of $\Gamma^{\mathrm{op}}$. The structure map associated with $\alpha: S \rightarrow T$ in $\Gamma^{\mathrm{op}}$ is the composite

$$
\operatorname{hocolim}_{H \mathcal{K}(S)} A_{S} \rightarrow \operatorname{hocolim}_{H \mathcal{K}(S)}\left(\alpha^{*}\left(A_{T}\right)\right) \rightarrow \operatorname{hocolim}_{H \mathcal{K}(T)} A_{T}
$$

induced by the map $\tilde{\alpha}$ and the map of homotopy colimits induced by $\alpha_{*}$.

The previous lemma ensures that $\gamma(A)$ has indeed the structure maps of a $\Gamma$-space. By definition, there is an isomorphism $\gamma(A)\left(1^{+}\right) \cong A_{h \mathcal{K}}$.

It is clear that $\gamma(A)$ is natural in $A$. Let $*$ be the terminal commutative $\mathcal{K}$-space monoid. Since $\operatorname{hocolim}_{\mathcal{K}}(*) \cong B \mathcal{K}$, it follows that $\gamma(*) \cong b \mathcal{K}$. We obtain:

Corollary 3.12 There is a canonical natural map $\gamma(A) \rightarrow b \mathcal{K}$ of $\Gamma$-spaces.

We have the following analogue of [26, Proposition 5.3]: 
Proposition 3.13 The $\Gamma$-space $\gamma(A)$ is special, and it is very special if $A$ is grouplike. For general $A$ there is a weak equivalence $B\left(A_{h \mathcal{K}}\right) \rightarrow \gamma(A)\left(S^{1}\right)=\gamma(A)(\mathbb{S})_{1}$.

Proof The proof of [26, Proposition 5.3] applies almost verbatim: the equivalence

$$
\gamma(A)(S)=\operatorname{hocolim}_{H \mathcal{K}(S)} A_{S} \rightarrow \operatorname{hocolim}_{\mathcal{K}^{\times \bar{S}}} A^{\times \bar{S}} \cong \prod_{\bar{S}} A_{h \mathcal{K}}
$$

shows that $\gamma(A)$ is special. It is easy to see that the condition of $A$ being grouplike and $\gamma(A)$ being very special refer to the same monoid structure on $\pi_{0}\left(A_{h \mathcal{K}}\right)$. The equivalence of the evaluation at the sphere $S^{1}$ with the bar construction follows as in [26, Proposition 5.3] from the choice of an ordering of the simplices in $S^{1}$.

The motivating examples for this construction come from the diagram space models for units of ring spectra introduced in the previous section.

Definition 3.14 Let $E$ be a commutative symmetric ring spectrum. The units of $E$ is the $\Gamma$-space $\mathrm{gl}_{1}^{\mathcal{I}} E=\gamma\left(\mathrm{GL}_{1}^{\mathcal{I}} E\right)$ associated with the commutative $\mathcal{I}$-space monoid $\mathrm{GL}_{1}^{\mathcal{I}} E$. The graded units is the $\Gamma$-space $\mathrm{gl}_{1}^{\mathcal{J}} E=\gamma\left(\mathrm{GL}_{1}^{\mathcal{J}} E\right)$ associated with the commutative $\mathcal{J}$-space monoid $\mathrm{GL}_{1}^{\mathcal{J}} E$. We view these constructions as functors

$$
\mathrm{gl}_{1}^{\mathcal{I}}: \mathcal{C} \operatorname{Sp}^{\Sigma} \rightarrow \Gamma^{\mathrm{op}}-\mathcal{S} \quad \text { and } \quad \mathrm{gl}_{1}^{\mathcal{J}}: \mathcal{C} \operatorname{Sp}^{\Sigma} \rightarrow \Gamma^{\mathrm{op}}-\mathcal{S} / b \mathcal{J}
$$

By construction, both $\mathrm{gl}_{1}^{\mathcal{I}} E$ and $\mathrm{gl}_{1}^{\mathcal{J}} E$ are very special. While the augmentation $\mathrm{gl}_{1}^{\mathcal{J}} E \rightarrow b \mathcal{J}$ will be central in what follows, we will usually ignore the augmentation of $\mathrm{gl}_{1}^{\mathcal{I}} E$ to the levelwise contractible $\Gamma$-space $b \mathcal{I}$. Both functors will only be homotopically well behaved for sufficiently fibrant (eg positive fibrant) $E$ since this was already the case with $\mathrm{GL}_{1}^{\mathcal{I}} E$ and $\mathrm{GL}_{1}^{\mathcal{J}} E$.

Remark 3.15 There is a classical notion of a spectrum of units associated with an $E_{\infty}$ spectrum due to May and coauthors [16]. The spectrum associated with $\mathrm{gl}_{1}^{\mathcal{I}} E$ is a possible construction of this object in the context of commutative symmetric ring spectra. It was introduced by Schlichtkrull [26]. Lind [12] shows that $\left(\mathrm{gl}_{1}^{\mathcal{I}} E\right)(\mathbb{S})$ is indeed equivalent to the spectra of units associated with other kinds of spectra.

We note that the $\Gamma$-spaces considered here are natural in the indexing category:

Lemma 3.16 Let $F: \mathcal{L} \rightarrow \mathcal{K}$ be a strong symmetric monoidal functor of small permutative categories and let $A$ be a commutative $\mathcal{K}$-space monoid. Then $F^{*}(A)$ is a commutative $\mathcal{L}$-space monoid, and there is a natural map $\gamma\left(F^{*} A\right) \rightarrow \gamma(A)$ of $\Gamma$-spaces. 


\section{Spectra of units}

Throughout this section we let $E$ be a positive fibrant commutative symmetric ring spectrum. We will compare the various spectra of units associated with $E$ and prove Theorem 1.2 from the introduction.

We begin by applying Lemma 3.16 and Lemma 2.12 to the units and the graded units of Definition 3.14 in order to obtain a sequence of $\Gamma$-spaces

$$
\mathrm{gl}_{1}^{\mathcal{I}} E \rightarrow \mathrm{gl}_{1}^{\mathcal{J}} E \rightarrow b \mathcal{J} \text {. }
$$

Proposition 4.1 The sequence (4-1) is a homotopy fiber sequence of $\Gamma$-spaces.

Remark 4.2 By definition, we call a sequence of $\Gamma$-spaces $W \rightarrow X \stackrel{g}{\rightarrow} Y$ a homotopy fiber sequence if $W$ maps by a stable equivalence into the base change of $\bar{g}$ along the map $* \rightarrow Y$, where $\bar{g}: \bar{X} \rightarrow Y$ is a replacement of $g$ by a stable fibration. All model category notions in this statement refer to the stable $Q$-model structure on $\Gamma$-spaces that we review in Section 5.

Some care is needed here because this model structure on $\Gamma$-spaces fails to be right proper (see Bousfield and Friedlander [7, Section 5.7]) and the above definition only provides a well-defined notion of homotopy fiber squares in a right proper model; see Goerss and Jardine [9, Section II.8]. There is no problem in the case at hand because all $\Gamma$-spaces in (4-1) are (up to a level fibrant replacement) stably fibrant. This is one reason why we do not try to replace the $\Gamma$-space $b \mathcal{J}$ by the stably equivalent $\Gamma$-space $\Gamma^{\mathrm{op}}\left(1^{+},-\right)$whose associated spectrum is the sphere spectrum $\left(S^{0}, S^{1}, \ldots\right)$.

Proof of Proposition 4.1 A general fact about left Bousfield localizations of model categories [10, Proposition 3.4.7] shows that a levelwise fibration between very special $\Gamma$-spaces is a fibration in the stable model structure on $\Gamma$-spaces. (This uses right properness of the level model structure, but not of the stable model structure.) So it is enough to show that the sequence (4-1) gives a homotopy fiber sequence of spaces when evaluated at any object $S$ of $\Gamma^{\mathrm{op}}$. Since all $\Gamma$-spaces involved are special, it is enough to check this for $S=1^{+}$.

Because $\mathrm{GL}_{1}^{\mathcal{J}} E$ is positive fibrant, [23, Lemma 4.12] (which is in turn based on [9, Lemma IV.5.7] leading to Quillen's Theorem B) shows that the commutative square

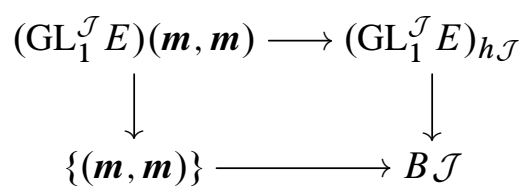


is homotopy cartesian if $m \geq 1$. Since $B \mathcal{I}$ is contractible, the same argument with $\mathcal{J}$ replaced by $\mathcal{I}$ shows that $\left(\mathrm{GL}_{1}^{\mathcal{I}} E\right)(\boldsymbol{m}) \rightarrow\left(\mathrm{GL}_{1}^{\mathcal{I}} E\right)_{h \mathcal{I}}$ is a weak equivalence. By Lemma 2.12, $\left(\mathrm{GL}_{1}^{\mathcal{J}} E\right)(\boldsymbol{m}, \boldsymbol{m}) \cong\left(\mathrm{GL}_{1}^{\mathcal{I}} E\right)(\boldsymbol{m})$, and the composite of the resulting map $\left(\mathrm{GL}_{1}^{\mathcal{J}} E\right)(\boldsymbol{m}, \boldsymbol{m}) \rightarrow\left(\mathrm{GL}_{1}^{\mathcal{I}} E\right)_{h \mathcal{I}}$ with the map $\left(\mathrm{GL}_{1}^{\mathcal{I}} E\right)_{h \mathcal{I}} \rightarrow\left(\mathrm{GL}_{1}^{\mathcal{J}} E\right)_{h \mathcal{J}}$ is the top horizontal map in (4-2). Hence $\left(\mathrm{GL}_{1}^{\mathcal{I}} E\right)_{h \mathcal{I}}$ maps by a weak equivalence into the homotopy fiber of $\left(\mathrm{GL}_{1}^{\mathcal{J}} E\right)_{h \mathcal{J}} \rightarrow B \mathcal{J}$ over $(\boldsymbol{m}, \boldsymbol{m})$. Since there is a morphism $(\mathbf{0}, \mathbf{0}) \rightarrow(\boldsymbol{m}, \boldsymbol{m})$ in $\mathcal{J}$, the same is true for $(\mathbf{0}, \mathbf{0})$ instead of $(\boldsymbol{m}, \boldsymbol{m})$. This verifies the claim because the object $(\mathbf{0 , 0})$ is the basepoint of $B \mathcal{J}$.

Definition 4.3 We define $\mathrm{bgl}_{1}^{*} E$ to be the homotopy cofiber of the map of spectra associated with the map of $\Gamma$-spaces $\mathrm{gl}_{1}^{\mathcal{J}} E \rightarrow b \mathcal{J}$.

This $\operatorname{bgl}_{1}^{*} E$ is an interesting object for the following reason: since the map $\pi_{0}\left(\mathrm{gl}_{1}^{\mathcal{J}} E\right) \rightarrow$ $\pi_{0}(b \mathcal{J}) \cong \pi_{0}(\mathbb{S}) \cong \mathbb{Z}$ is in general not surjective, the homotopy fiber sequence (4-1) does not necessarily induce a homotopy fiber sequence of spectra. If it gave a homotopy fiber sequence of spectra, the spectrum $\operatorname{bgl}_{1}^{*} E$ would simply be the suspension of the spectrum $\left(\mathrm{gl}_{1}^{\mathcal{I}} E\right)(\mathbb{S})$ associated with $\mathrm{gl}_{1}^{\mathcal{I}} E$. This suspension is usually denoted by $\operatorname{bgl}_{1} E$.

The failure of preserving homotopy fiber sequences means that $\operatorname{bgl}_{1}^{*} E$ and $\operatorname{bgl}_{1} E$ differ in general. The point of Theorem 1.2 is that the "graded" version $\operatorname{bgl}_{1}^{*} E$ defined here extends the usual $\operatorname{bgl}_{1} E$ in an interesting way.

Proof of Theorem 1.2 Since the spectra associated with $\mathrm{gl}_{1}^{\mathcal{J}} E$ and $b \mathcal{J}$ are connective, $\operatorname{bgl}_{1}^{*} E$ is connective. The right adjoint of $X \mapsto X(\mathbb{S})$ is a right Quillen functor and may be viewed as a model for the connective cover; see Bousfield and Friedlander [7, Section 5]. Rewriting the homotopy cofiber sequence defining $\operatorname{bgl} 1_{1}^{*} E$ as a homotopy fiber sequence of spectra

$$
\Omega\left(\mathrm{bgl}_{1}^{*} E\right) \rightarrow\left(\mathrm{gl}_{1}^{\mathcal{J}} E\right)(\mathbb{S}) \rightarrow(b \mathcal{J})(\mathbb{S}),
$$

we can apply the right adjoint and compare with the homotopy fiber sequence (4-1) to see that the connective cover of $\Omega\left(\operatorname{bgl}_{1}^{*} E\right)$ has the homotopy type of $\left(\mathrm{gl}_{1}^{\mathcal{I}} E\right)(\mathbb{S})$. Proposition 4.6 below verifies the claim about $\pi_{0}\left(\mathrm{bgl}_{1}^{*} E\right)$.

Remark 4.4 One may also formulate Theorem 1.2 in terms of the homotopy fiber $\mathrm{gl}_{1}^{*} E \simeq \Omega\left(\operatorname{bgl}_{1}^{*} E\right)$ of the map of spectra associated with $\mathrm{gl}_{1}^{\mathcal{J}} E \rightarrow b \mathcal{J}$ by saying that $\mathrm{gl}_{1}^{*} E$ is a $(-2)$-connected spectrum with $\left(\mathrm{gl}_{1}^{\mathcal{I}} E\right)(\mathbb{S})$ as its connective cover. Another reformulation is to say that $\operatorname{bgl}_{1} E$ is the 0 -connected cover of $\mathrm{bgl}_{1}^{*} E$.

We recall the notion of periodicity from Theorem 1.2: 
Definition 4.5 The periodicity $n_{E} \in \mathbb{N}_{0}$ of $E$ is defined to be zero if all units in the underlying multiplicative graded monoid of $\pi_{*}(E)$ have degree 0 . Otherwise $n_{E}$ is the minimal positive integer such that $\pi_{*}(E)$ has a unit of degree $n_{E}$.

Proposition 4.6 There is an isomorphism of exact sequences:

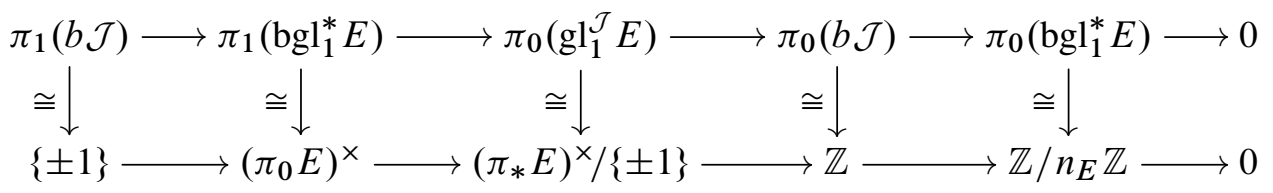

Proof By definition of the $\Gamma$-space $\mathrm{gl}_{1}^{\mathcal{J}} E$ we know $\pi_{0}\left(\mathrm{gl}_{1}^{\mathcal{J}} E\right) \cong \pi_{0}\left(\mathrm{GL}_{1}^{\mathcal{J}} E\right)_{h \mathcal{J}}$. The latter group is isomorphic to $\pi_{*}(E)^{\times} /\{ \pm 1\}$ by [23, Corollary 4.17] and [23, Proposition 4.26]. The image of the map from $\pi_{0}\left(\mathrm{gl}_{1}^{\mathcal{J}} E\right)$ to $\pi_{0}(b \mathcal{J}) \cong \pi_{0}(\mathbb{Z}) \cong \mathbb{Z}$ is $n_{E} \mathbb{Z}$ since it is the set of all integers that arise as the degree of a unit in the graded monoid $\pi_{*}(E)$. Hence $\pi_{0}\left(\operatorname{bgl} 1_{1}^{*} E\right) \cong \mathbb{Z} / n_{E} \mathbb{Z}$.

Since $\left(\mathrm{gl}_{1}^{\mathcal{I}} E\right)(\mathbb{S})$ is the connective cover of $\Omega\left(\operatorname{bgl}_{1}^{*} E\right)$, we obtain isomorphisms $\pi_{1}\left(\operatorname{bgl}_{1}^{*} E\right) \cong \pi_{0}\left(\mathrm{gl}_{1}^{\mathcal{I}} E\right) \cong \pi_{0}(E)^{\times}$. The action of $\pi_{1}(b \mathcal{J}) \cong \pi_{1}(\mathbb{S}) \cong \mathbb{Z} / 2$ is identified with the sign action in [23, Proposition 4.24]

Remark 4.7 The proposition implies that $\mathrm{bgl}_{1}^{*}$ detects periodicity: If $E$ is periodic and $e \rightarrow E$ is its connective cover, $\pi_{0}\left(\operatorname{bgl}_{1}^{*}(e \rightarrow E)\right)$ is the surjection $\mathbb{Z} \rightarrow \mathbb{Z} / n_{E} \mathbb{Z}$. In contrast, the map $\mathrm{gl}_{1}^{\mathcal{I}} e \rightarrow \mathrm{gl}_{1}^{\mathcal{I}} E$ is a stable equivalence. Its associated map of spectra is equivalent to the connective cover of $\Omega \operatorname{bgl}_{1}^{*}(e \rightarrow E)$.

Remark 4.8 Proposition 4.6 also shows that the stable Hopf map (as a generator of $\left.\pi_{1}(\mathbb{S})\right)$ gives rise to the sign action on $\left(\pi_{0} E\right)^{\times}:$On $\pi_{1}$, the map $\mathbb{S} \rightarrow \operatorname{bgl} 1_{1}^{*} E$ sends the generator to $-1 \in\left(\pi_{0} E\right)^{\times}$. This relation is not visible in the ordinary $\operatorname{bgl}_{1} E$.

In most cases, $\operatorname{bgl}_{1}^{*} E$ is not just a product of $\operatorname{bgl}_{1} E$ with an Eilenberg-MacLane spectrum on $\mathbb{Z} / n_{E} \mathbb{Z}$ :

Lemma 4.9 If the action by $\{ \pm 1\}$ on the group $\pi_{0}(E)^{\times}$induced by the additive structure of the ring $\pi_{0}(E)$ is non-trivial, then the spectrum $\operatorname{bgl}_{1}^{*} E$ has a non-vanishing first $k$-invariant.

Proof If the first $k$-invariant of $\operatorname{bgl}_{1}^{*} E$ vanishes, the Postnikov section $P_{1}\left(\mathrm{bgl}_{1}^{*} E\right)$ decomposes as the product of suspended Eilenberg-MacLane spectra. In view of Proposition 4.6, this is a contradiction because the map from $\mathbb{S}$ to $P_{1}\left(\mathrm{bgl}_{1}^{*} E\right)$ that is the surjection $\mathbb{Z} \rightarrow \mathbb{Z} / n_{E} \mathbb{Z}$ on $\pi_{0}$ is non-trivial on $\pi_{1}$ by assumption. 
Example 4.10 The last lemma applies to the periodic complex $K$-theory spectrum KU. We have $\pi_{0} \mathrm{bgl}_{1}^{*} \mathrm{KU} \cong \mathbb{Z} / 2 \mathbb{Z}$ and $\pi_{1} \mathrm{bgl}_{1}^{*} \mathrm{KU} \cong \pi_{0}(\mathrm{KU})^{\times} \cong \mathbb{Z} / 2 \mathbb{Z}$. So the first $k-$ invariant of $\operatorname{bgl}_{1}^{*} \mathrm{KU}$ is a non-zero element of $H^{2}(H \mathbb{Z} / 2 ; \mathbb{Z} / 2)$ and hence equals $\mathrm{Sq}^{2}$.

Remark 4.11 We give a brief sketch of how the map $\mathbb{S} \rightarrow \mathrm{bgl}_{1}^{*} E$ resulting from the construction of $\operatorname{bgl}_{1}^{*} E$ can be understood in terms of more well-known maps. A detailed account of this will be included in forthcoming joint work with Schlichtkrull [22]. Naturality of $\mathrm{gl}_{1}^{\mathcal{J}}$ implies that $\mathbb{S} \rightarrow \operatorname{bgl}_{1}^{*} E$ factors as the composite of $\mathbb{S} \rightarrow \operatorname{bgl}_{1}^{*} \mathbb{S}$ and the map $\operatorname{bgl}_{1}^{*} \mathbb{S} \rightarrow \operatorname{bgl}_{1}^{*} E$ induced by $\mathbb{S} \rightarrow E$. So we may restrict to the case $E=\mathbb{S}$.

Let $\mathcal{W}=\mathcal{O}^{-1} \mathcal{O}$ be Quillen's localization construction on the category $\mathcal{O}$ of standard inner product spaces $\mathbb{R}^{n}$ and linear isometric isomorphisms. The category $\mathcal{W}$ is the orthogonal counterpart of $\mathcal{J}$ and is studied in joint work with Schlichtkrull [25]. It is related to orthogonal spectra in the same way as $\mathcal{J}$ is related to symmetric spectra, and to the orthogonal version of $\mathcal{I}$-spaces studied by Lind [12] in the same way as $\mathcal{J}$ is related to $\mathcal{I}$. (The $\mathcal{W}$-spaces considered here and in [25] should not be confused with the continuous functors on based spaces homeomorphic to finite $\mathrm{CW}$-complexes studied under this name elsewhere; see eg Mandell, May, Schwede and Shipley [14, Example 4.6].)

Since $\mathbb{S}$ can be viewed as an orthogonal spectrum, it has an associated commutative $\mathcal{W}$-space monoid of units and an associated $\Gamma$-space $\operatorname{gl}_{1}^{\mathcal{W}} \mathbb{S}$ augmented over $b \mathcal{W}$. The cofiber of the map of spectra induced by the augmentation is equivalent to $\operatorname{bgl}_{1}^{*} E$, and it follows that $\mathbb{S} \rightarrow \operatorname{bgl}_{1}^{*} \mathbb{S}$ factors as the map $\mathbb{S} \simeq b \mathcal{J} \rightarrow b \mathcal{W} \simeq$ ko induced by the unit and a map ko $\simeq b \mathcal{W} \rightarrow \operatorname{bgl}_{1}^{*} \mathbb{S}$. The underlying $E_{\infty}$ space of the 0-connected cover of ko is BO, the underlying $E_{\infty}$ space of the 0 -connected cover $\operatorname{bgl}_{1} \mathbb{S}$ of $\operatorname{bgl}_{1}^{*} \mathbb{S}$ is $\mathrm{BF}$, and the map of underlying $E_{\infty}$ spaces of the 0 -connected covers is the stable $J$-homomorphism BO $\rightarrow$ BF.

\section{Grouplike graded commutative spaces}

In Section 3 we observed that a grouplike commutative $\mathcal{J}$-space monoid $A$ gives rise to a map of very special $\Gamma$-spaces $\gamma(A) \rightarrow b \mathcal{J}$. In this section we explain how this construction induces an equivalence between the homotopy categories of grouplike commutative $\mathcal{J}$-space monoids and $\Gamma$-spaces over the $\Gamma$-space $b \mathcal{J}$ and why this is relevant for the graded units $\mathrm{gl}_{1}^{\mathcal{J}}$.

We start by recalling the definition of the positive $\mathcal{J}$-model structure on commutative $\mathcal{J}$ space monoids from [23, Section 4]. The weak equivalences are the $\mathcal{J}$-equivalences of Definition 2.3, that is, the maps which induce weak equivalences on homotopy colimits 
over $\mathcal{J}$. A map $f: A \rightarrow B$ in $\mathcal{C S}^{\mathcal{J}}$ is a positive $\mathcal{J}$-fibration if every morphism $\left(\beta_{1}, \beta_{2}, \sigma\right):\left(\boldsymbol{m}_{\mathbf{1}}, \boldsymbol{m}_{\mathbf{2}}\right) \rightarrow\left(\boldsymbol{n}_{\mathbf{1}}, \boldsymbol{n}_{\mathbf{2}}\right)$ in $\mathcal{J}$ with $m_{1} \geq 1$ induces a homotopy cartesian square

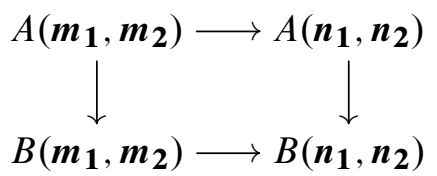

in which the vertical maps are Kan fibrations. The positive $\mathcal{J}$-cofibrations are determined by a lifting property. It is shown in [23, Proposition 4.10] that these classes of maps form a (cofibrantly generated proper) model structure on $\mathcal{C S}^{\mathcal{J}}$. The analogy to $\mathcal{I}$-spaces discussed in the introduction and the following two results indicate that this model structure gives an interesting homotopy theory of commutative $\mathcal{J}$-space monoids.

Proposition 5.1 [23, Proposition 4.23] There is a Quillen adjunction

$$
\mathbb{S}^{\mathcal{J}}[-]: \mathcal{C} \mathcal{S}^{\mathcal{J}} \rightleftarrows \mathcal{C} \operatorname{Sp}^{\Sigma}: \Omega^{\mathcal{J}}
$$

with respect to the positive $\mathcal{J}$-model structure and the positive stable model structure on the category of commutative symmetric ring spectra introduced by Mandell, May, Schwede and Shipley [14].

Theorem 5.2 [23, Theorem 1.7] There is a chain of Quillen equivalences between the positive $\mathcal{J}$-model structure on $\mathcal{C S}^{\mathcal{J}}$ and the category of $E_{\infty}$ spaces over $B \mathcal{J}$.

In the theorem, $E_{\infty}$ spaces are spaces with an action of the Barratt-Eccles operad. It uses that the latter acts on the classifying space of a permutative category.

\subsection{Group completion for commutative $\mathcal{J}$-space monoids}

The recognition principle for infinite loop spaces states that grouplike $E_{\infty}$ spaces are equivalent to connective spectra; see Boardman and Vogt [5] and May [15]. Together with the last theorem, this suggests that grouplike commutative $\mathcal{J}$-space monoids should be equivalent to connective spectra over the spectrum associated with the permutative category $\mathcal{J}$.

We will prove this by first building a model category that is closely related to group completion and whose homotopy category is equivalent to the subcategory of grouplike objects in $\operatorname{Ho}\left(\mathcal{C S}^{\mathcal{J}}\right)$. In a second step, we show that there is a chain of Quillen equivalences relating this model category to the category of $\Gamma$-spaces over $b \mathcal{J}$ with a stable model structure. This implies the above statement about the equivalence of the 
respective homotopy categories. At the same time, it shows the much stronger statement that homotopy classes of maps and the homotopy types of mapping spaces coincide.

A similar description of the classical recognition principle in terms of Quillen equivalences is given in [24]. Working in the context of commutative $\mathcal{I}$-space monoids, it is even possible to build a single Quillen equivalence inducing an equivalence between the homotopy category of grouplike commutative $\mathcal{I}$-space monoids and the homotopy category of connective spectra.

In the following we use that the homotopy colimit $A_{h \mathcal{J}}$ associated with a commutative $\mathcal{J}$-space monoid is a simplicial monoid so that we can form the bar construction $B\left(A_{h \mathcal{J}}\right)=B\left(*, A_{h \mathcal{J}}, *\right)$.

Definition 5.4 (i) A commutative $\mathcal{J}$-space monoid $A$ is grouplike if the commutative monoid $\pi_{0}\left(A_{h \mathcal{J}}\right)$ is a group.

(ii) A map of commutative $\mathcal{J}$-space monoids $A \rightarrow A^{\prime}$ is a group completion if $B\left(A_{h \mathcal{J}}\right) \rightarrow B\left(A_{h \mathcal{J}}^{\prime}\right)$ is a weak equivalence of spaces and $A^{\prime}$ is grouplike.

(iii) A map $A \rightarrow A^{\prime}$ in $\mathcal{C} \mathcal{S}^{\mathcal{J}}$ induces an equivalence after group completion if the induced map of bar constructions $B\left(A_{h \mathcal{J}}\right) \rightarrow B\left(A_{h \mathcal{J}}^{\prime}\right)$ is a weak equivalence.

Following the usual terminology that a map of $\Gamma$-spaces is a stable equivalence if it induces a stable equivalence of associated spectra, Proposition 3.13 implies that $A \rightarrow A^{\prime}$ induces an equivalence after group completion if and only if $\gamma(A) \rightarrow \gamma\left(A^{\prime}\right)$ is a stable equivalence. We note that the existence of group completions in $\mathcal{C S}^{\mathcal{J}}$ is not needed for this notion of equivalence.

Theorem 5.5 The category of commutative $\mathcal{J}$-space monoids $\mathcal{C S}^{\mathcal{J}}$ admits a model structure in which the weak equivalences are the maps that induce weak equivalences after group completion and the cofibrations are the positive $\mathcal{J}$-cofibrations.

A commutative $\mathcal{J}$-space monoid is fibrant in this model structure if and only if it is positive $\mathcal{J}$-fibrant and grouplike. The fibrant replacement is a group completion in the sense of Definition 5.4. Fibrations are characterized by the right lifting property.

We refer to this model structure as the group completion model structure and write $\mathcal{C S}_{\text {gp }}^{\mathcal{J}}$ for this model category. Its homotopy category is the homotopy category of grouplike commutative $\mathcal{J}$-space monoids.

The model structure of Theorem 5.5 will be constructed by localizing the positive $\mathcal{J}$-model structure with respect to an explicit set of "shear maps" to be defined in (7-6). The difficult part is to identify the weak equivalences and fibrant objects. We prove the theorem in Section 7.13. 
Remark 5.6 A similar group completion model structure for commutative $\mathcal{I}$-space monoids has been developed in [24]. A key ingredient for the $\mathcal{I}$-space result was the fact that the ordinary group completion of simplicial monoids lifts to commutative $\mathcal{I}$-space monoids: one can define a bar construction $B^{\bigotimes}$ and a loop functor $\Omega$ so that $A \rightarrow \Omega B^{\bigotimes}(A)$ is a group completion for the commutative $\mathcal{I}$-space monoid $A$. This does not carry over to commutative $\mathcal{J}$-space monoids: since $\mathcal{C} \mathcal{S}^{\mathcal{J}}$ has no zero object, there is no loop functor on $\mathcal{C S}^{\mathcal{J}}$ that serves for the above purpose. (The point is that already for ordinary $\mathbb{Z}$-graded commutative monoids, the initial object is concentrated in degree 0 while the terminal object is non-empty in every degree.)

In particular, the fibrant replacement in the above model structure is the only construction of a group completion functor for commutative $\mathcal{J}$-space monoids we are currently aware of.

Remark 5.7 As discussed in Section 1.8, Theorem 5.5 is used in an essential way for the study of ring spectra with graded logarithmic structures and their logarithmic topological André-Quillen homology and logarithmic topological Hochschild homology. We refer to our joint work with Rognes and Schlichtkrull $[21 ; 19 ; 20]$ for details of these applications.

Example 5.8 We analyze the group completion of commutative $\mathcal{J}$-space monoids in an example. Let

$$
A=\mathbb{C} F_{\left(\boldsymbol{m}_{\mathbf{1}}, \boldsymbol{m}_{\mathbf{2}}\right)}^{\mathcal{J}}(*) \cong \coprod_{n \geq 0}\left(F_{\left(\boldsymbol{m}_{\mathbf{1}}, \boldsymbol{m}_{\mathbf{2}}\right)}^{\mathcal{J}}(*)\right)^{\otimes n} / \Sigma_{n}
$$

be the free commutative $\mathcal{J}$-space monoid on a point in degree $\left(\boldsymbol{m}_{\mathbf{1}}, \boldsymbol{m}_{\mathbf{2}}\right)$. We assume $m_{1} \geq 1$ to ensure that the symmetric group action in the definition of $A$ is free. It follows that

$$
A_{h \mathcal{J}} \simeq \coprod_{n \geq 0} B \Sigma_{n}
$$

Let $A \rightarrow A^{\text {gp }}$ be a fibrant replacement in the group completion model structure. The Barratt-Priddy-Quillen theorem implies that $A_{h \mathcal{J}} \rightarrow\left(A^{\mathrm{gp}}\right)_{h \mathcal{J}}$ has the homotopy type of the group completion map $\bigsqcup_{n \geq 0} B \Sigma_{n} \rightarrow Q S^{0}$.

The map $A^{\mathrm{gp}} \rightarrow *$ induces an augmentation $Q S^{0} \simeq\left(A^{\mathrm{gp}}\right)_{h \mathcal{J}} \rightarrow B \mathcal{J} \simeq Q S^{0}$. The homotopy class of this map is determined by the image of a generator under the induced map of path components. Since the generator of $\pi_{0}\left(A_{h \mathcal{J}}\right) \cong \mathbb{N}$ is mapped to $m_{2}-m_{1}$ in $\pi_{0}(B \mathcal{J}) \cong \mathbb{Z}$, it follows that $\left(A^{\mathrm{gp}}\right)_{h \mathcal{J}} \rightarrow B \mathcal{J}$ is multiplication by $m_{2}-m_{1}$. This implies that in the special case $m_{2}-m_{1}=1$, the group completion $A^{\text {gp }}$ is particularly simple: the map $A^{\mathrm{gp}} \rightarrow *$ is a $\mathcal{J}$-equivalence. 


\subsection{Grouplike commutative $\mathcal{J}$-space monoids and connective spectra}

In order to give the characterization of grouplike commutative $\mathcal{J}$-space monoids outlined above, we will again use $\Gamma$-spaces as a model for connective spectra. The category $\Gamma^{\mathrm{op}}-\mathcal{S}$ admits a stable Quillen model structure (stable Q-model structure for short) whose homotopy category is the homotopy category of connective spectra; see Schwede [27] and Bousfield and Friedlander [7]. We write $\left(\Gamma^{\mathrm{op}}-\mathcal{S}\right)_{\mathrm{st}}$ for this model category. It will be reviewed in Section 7.13.

We will use that for an object $Z$ in a model category $\mathcal{M}$, the comma category $\mathcal{M} \downarrow Z$ of objects over $Z$ inherits a "overcategory" model structure in which a map is a weak equivalence, cofibration or fibration if its projection to $\mathcal{M}$ is; see Hirschhorn [10, Section 7.6].

Theorem 5.10 There is a chain of Quillen equivalences relating $\mathcal{C S}^{\mathcal{J}}$ with the group completion model structure and the category $\left(\Gamma^{\mathrm{op}}-\mathcal{S}\right)_{\mathrm{st}} / b \mathcal{J}$.

The chain of Quillen equivalences can be chosen so that the value of the composed derived functor $\mathcal{C S}^{\mathcal{J}} \rightarrow\left(\Gamma^{\mathrm{op}}-\mathcal{S}\right) / b \mathcal{J}$ on a commutative $\mathcal{J}$-space monoid $A$ and the explicit $\Gamma$-space $\gamma(A)$ of Definition 3.11 are stably equivalent over $b \mathcal{J}$.

Here the derived functor of a left (or right) Quillen functor means the functor of model categories obtained by precomposing it with a cofibrant (or fibrant) replacement functor. The composed derived functor is the composite of the derived functors associated with the Quillen functors in the zig-zag of Quillen equivalences.

The proof of Theorem 5.10 and the construction of the intermediate model categories that are implicit in its formulation will be given in Section 7. Theorem 5.10 and Theorem 5.5 combine to give Theorem 1.6 from the introduction.

\subsection{Graded units of ring spectra as a right adjoint}

We earlier defined the units $A^{\times}$of a commutative $\mathcal{J}$-space monoid $A$ as the sub commutative $\mathcal{J}$-space monoid of invertible path components. They come with a natural map $A^{\times} \rightarrow A$. This construction admits a useful formulation in terms of a model structure which is somewhat dual to the group completions discussed in Section 5.3:

Theorem 5.12 The category of commutative $\mathcal{J}$-space monoids $\mathcal{C} \mathcal{S}^{\mathcal{J}}$ admits a model structure in which a map $f: A \rightarrow B$ is a weak equivalence if the induced map $f^{\times}: A^{\times} \rightarrow B^{\times}$is a $\mathcal{J}$-equivalence. The fibrations are the positive $\mathcal{J}$-fibrations. 
The cofibrant objects are the commutative $\mathcal{J}$-space monoids which are positive $\mathcal{J}$ cofibrant and grouplike, and the cofibrant replacement of $A$ is $\mathcal{J}$-equivalent to $A^{\times} \rightarrow A$. The general cofibrations are determined by a lifting property.

We call the resulting model structure $\mathcal{C} \mathcal{S}_{\text {un }}^{\mathcal{J}}$ the units model structure. The proof of the theorem is completely analogous to the proof of the corresponding statement about commutative $\mathcal{I}$-space monoids in [24, Theorem 1.8] and will not be repeated here.

The identity functors form a Quillen adjunction $\mathcal{C S}_{\text {un }}^{\mathcal{J}} \rightleftarrows \mathcal{C S}^{\mathcal{J}}$ (where as always in this paper the left adjoint is the upper arrow). Together with the Quillen adjunction $\mathcal{C S}^{\mathcal{J}} \rightleftarrows \mathcal{C S}_{\text {gp }}^{\mathcal{J}}$ coming from the group completion model structure, we obtain:

Corollary 5.13 The identity functors form a Quillen equivalence $\mathcal{C} \mathcal{S}_{\text {un }}^{\mathcal{J}} \rightleftarrows \mathcal{C S}_{\text {gp }}^{\mathcal{J}}$.

Proof On both sides, a weak equivalence from a positive $\mathcal{J}$-cofibrant and grouplike object into a positive $\mathcal{J}$-fibrant and grouplike object is a $\mathcal{J}$-equivalence.

Combining this with the Quillen adjunction of Proposition 5.1, we get a chain of Quillen adjunctions

$$
\mathcal{C S p}{ }^{\Sigma} \leftrightarrows \mathcal{C} \mathcal{S}^{\mathcal{J}} \leftrightarrows \mathcal{C} \mathcal{S}_{\text {un }}^{\mathcal{J}} \rightleftarrows \mathcal{C} \mathcal{S}_{\mathrm{gp}}^{\mathcal{J}}
$$

in which the last one is a Quillen equivalence. We can use this to identify $\mathrm{gl}_{1}^{\mathcal{J}}$ as a right adjoint on the level of homotopy categories:

Proof of Theorem 1.7 For a positive fibrant commutative symmetric ring spectrum, Theorem 5.12 implies that the units $\mathrm{GL}_{1}^{\mathcal{J}} E$ are $\mathcal{J}$-equivalent to the cofibrant replacement of $\Omega^{\mathcal{J}} E$ in $\mathcal{C} \mathcal{S}_{\text {un }}^{\mathcal{J}}$. Together with Theorem 5.10, the chain of adjunctions (5-2) implies that $\mathrm{gl}_{1}^{\mathcal{J}} E=\gamma\left(\mathrm{GL}_{1}^{\mathcal{J}} E\right)$ is as an augmented object stably equivalent to the composed derived functor $\mathcal{C} \operatorname{Sp}^{\Sigma} \rightarrow\left(\Gamma^{\mathrm{op}}-\mathcal{S}\right) / b \mathcal{J}$ of a zig-zag of Quillen adjunctions.

Right Quillen functors induce right adjoints of homotopy categories. Left Quillen functors that participate in Quillen equivalences induce equivalences of homotopy categories, which are in particular right adjoints. Since all left Quillen functors in the zig-zag of Quillen adjunctions describing $\mathrm{gl}_{1}^{\mathcal{J}}$ are part of a Quillen equivalence, the claim follows.

Remark 5.14 Since the equivalences of homotopy categories arise from inverting Quillen equivalences, the previous proof shows more than is stated in the theorem: the adjunction $\operatorname{Ho}\left(\mathcal{C S p}^{\Sigma}\right) \leftrightarrows \operatorname{Ho}\left(\left(\Gamma^{\mathrm{op}}-\mathcal{S}\right)_{\mathrm{st}} / b \mathcal{J}\right)$ induced by $\mathrm{gl}_{1}^{\mathcal{J}}$ is compatible with the homotopy types of mapping spaces on both sides. 


\section{Gamma- $\mathcal{J}$-spaces}

In this section we construct the zig-zag of adjunctions that will be used in the proofs of Theorem 5.5 and Theorem 5.10, and we make the first step towards the model structures that appear in the theorems.

Let $F: \mathcal{C} \rightarrow$ CAT be a functor to the category of small categories. Its Grothendieck construction is the category $\mathcal{C} \int F$ whose objects are pairs $(C, X)$ with $C \in \mathrm{Ob}(\mathcal{C})$ and $X \in \mathrm{Ob}(F(C))$. A morphism $(\alpha, f):(C, X) \rightarrow(D, Y)$ consists of a morphism $\alpha: C \rightarrow D$ in $\mathcal{C}$ and a morphism $f: F(\alpha)(X) \rightarrow Y$ in $F(D)$. Its composite with $(\beta, g):(D, Y) \rightarrow(E, Z)$ is $(\beta \alpha, g(F(\beta)(f)))$.

The next definition refers to the functor $H \mathcal{J}: \Gamma^{\mathrm{op}} \rightarrow$ CAT defined in Section 3.2.

Definition 6.1 We let $\Gamma^{\mathrm{op}} \mathcal{J}$ be the Grothendieck construction $\Gamma^{\mathrm{op}} \int H \mathcal{J}$ on $H \mathcal{J}$.

Objects of $\Gamma^{\mathrm{op}} \mathcal{J}$ are tuples $(S ; \boldsymbol{s}, \sigma)$ with $S \in \mathrm{Ob}\left(\Gamma^{\mathrm{op}}\right)$ a finite based set and $(\boldsymbol{s}, \sigma)$ an object of the category $H \mathcal{J}(S)$. The object $\left(0^{+} ; \mathbf{0}_{0^{+}}\right)$is terminal in $\Gamma^{\mathrm{op}} \mathcal{J}$. (It is not initial because not every object in $\mathcal{J}$ receives a map from $(\mathbf{0}, \mathbf{0})$.)

Definition 6.2 Let $\Gamma^{\mathrm{op}} \mathcal{J}-\mathcal{S}$ be the category of functors $X: \Gamma^{\mathrm{op}} \mathcal{J} \rightarrow \mathcal{S}$ that send $\left(0^{+} ; \mathbf{0}_{0^{+}}\right)$to the one-point space.

Example 6.3 For $(\boldsymbol{s}, \sigma) \in \mathrm{Ob}(H \mathcal{J}(S))$, the functor $\Gamma^{\mathrm{op}} \mathcal{J}((S ; \boldsymbol{s}, \sigma),-)$ : $\Gamma^{\mathrm{op}} \mathcal{J} \rightarrow \mathcal{S}$ defines a $\Gamma^{\mathrm{op}} \mathcal{J}$-space since $\left(0^{+}, \mathbf{0}_{0^{+}}\right)$is terminal.

The category $\Gamma^{\mathrm{op}} \mathcal{J}-\mathcal{S}$ admits an equivalent description that will provide us with more examples. For this we recall from Section 3.9 that $\alpha: S \rightarrow T$ in $\Gamma^{\text {op }}$ induces a functor $\alpha_{*}: H \mathcal{J}(S) \rightarrow H \mathcal{J}(T)$ and hence $\alpha^{*}: \mathcal{S}^{H \mathcal{J}(T)} \rightarrow \mathcal{S}^{H \mathcal{J}(S)}$.

Lemma 6.4 $A \Gamma^{\mathrm{op}} \mathcal{J}$-space is the same as a collection of $H \mathcal{J}(S)$-spaces $X_{S}$ for every finite based set $S$ and maps of $H \mathcal{J}(S)$-spaces $\tilde{\alpha}: X_{S} \rightarrow \alpha^{*}\left(X_{T}\right)$ for every map $\alpha: S \rightarrow T$ in $\Gamma^{\mathrm{op}}$ such that $X_{0^{+}}=*$ and $\left(\alpha^{*}(\widetilde{\beta})\right) \widetilde{\alpha}=\widetilde{\beta \alpha}$ holds for composable maps in $\Gamma^{\mathrm{op}}$. Morphisms of $\Gamma^{\mathrm{op}} \mathcal{J}$-spaces correspond to families of maps of $H \mathcal{J}(S)$-spaces $f_{S}: X_{S} \rightarrow Y_{S}$ such that $\left(\alpha^{*}\left(f_{T}\right)\right) \tilde{\alpha}=\tilde{\alpha} f_{S}$.

Proof If $X$ is a $\Gamma^{\mathrm{op}} \mathcal{J}$-space, then the $X_{S}=X(S ;-)$ have this property. The other direction follows from the definition of the Grothendieck construction.

The lemma applies to the $H \mathcal{J}(S)$-spaces $A_{S}$ and maps $\tilde{\alpha}$ defined in Section 3.9: 
Corollary 6.5 The collection of $H \mathcal{J}(S)$-spaces $A_{S}$ associated with a commutative $\mathcal{J}$-space monoid $A$ defines a $\Gamma^{\mathrm{op}} \mathcal{J}$-space.

Colimits in $\Gamma^{\mathrm{op}} \mathcal{J}-\mathcal{S}$ are not the levelwise colimits of the underlying $H \mathcal{J}(S)$-spaces because this would violate the condition on $\left(0^{+}, \mathbf{0}_{0^{+}}\right)$. To construct them, we note that the basepoint condition on a $\Gamma^{\mathrm{op}} \mathcal{J}$-space $X$ induces a canonical map

$$
\Gamma^{\mathrm{op}} \mathcal{J}\left(\left(0^{+} ; \mathbf{0}_{0^{+}}\right),-\right) \rightarrow X .
$$

Hence $\Gamma^{\mathrm{op}} \mathcal{J}-\mathcal{S}$ may be viewed as a full subcategory of $\Gamma^{\mathrm{op}} \mathcal{J}\left(\left(0^{+}, \mathbf{0}_{\mathbf{0}^{+}}\right),-\right) \downarrow \mathcal{S}^{\Gamma^{\mathrm{op}} \mathcal{J}}$, and the colimits and limits in the larger category exist and provide colimits and limits in $\Gamma^{\text {op }} \mathcal{J}-\mathcal{S}$. This proves the next lemma.

Lemma 6.6 The category $\Gamma^{\mathrm{op}} \mathcal{J}-\mathcal{S}$ is complete and cocomplete.

Let $X$ and $Y$ be $\Gamma^{\text {op }} \mathcal{J}$-spaces and $K$ a simplicial set. We define $X \otimes K$ to be the $\Gamma^{\mathrm{op}} \mathcal{J}$-space given by the pushout

$$
\Gamma^{\mathrm{op}} \mathcal{J}\left(\left(0^{+}, \mathbf{0}_{0^{+}}\right),-\right) \leftarrow \Gamma^{\mathrm{op}} \mathcal{J}\left(\left(0^{+}, \mathbf{0}_{0^{+}}\right),-\right) \times K \rightarrow X \times K
$$

in $\mathcal{S}^{\Gamma^{\mathrm{op}} \mathcal{J}}$. Here $-\times K$ denotes the objectwise product with $K$. Moreover, we define a $\Gamma^{\text {op }} \mathcal{J}$-space $X^{K}$ by $X^{K}(S ; s, \sigma)=(X(S ; s, \sigma))^{K}$ and a simplicial set $\operatorname{Map}(X, Y)$ by $[m] \mapsto \Gamma^{\text {op }} \mathcal{J}-\mathcal{S}\left(X \otimes \Delta^{m}, Y\right)$. Using Goerss and Jardine [9, II Lemma 2.4] we conclude the following statement.

Proposition 6.7 With these definitions, $\Gamma^{\mathrm{op}} \mathcal{J}-\mathcal{S}$ is tensored, cotensored and enriched over unpointed simplicial sets.

For the construction of model structures on $\Gamma^{\mathrm{op}} \mathcal{J}-\mathcal{S}$ we will need certain free functors that we introduce next. Let $X$ be a $\Gamma^{\mathrm{op}} \mathcal{J}$-space and let $X_{S}$ be the associated $H \mathcal{J}(S)$ space. The unique map $\alpha: 0^{+} \rightarrow S$ induces a map

$$
*=X_{0^{+}}\left(\mathbf{0}_{0^{+}}\right) \rightarrow \alpha^{*}(X)\left(\mathbf{0}_{0^{+}}\right)=X_{S}\left(\mathbf{0}_{S}\right)
$$

that makes $X_{S}\left(\mathbf{0}_{S}\right)$ a pointed space and $X_{S}$ an $H \mathcal{J}(S)$-space under the free $H \mathcal{J}(S)$ space $H \mathcal{J}(S)\left(\mathbf{0}_{S},-\right)$. By slight abuse of notation, we write $\mathcal{S}_{*}^{H \mathcal{J}(S)}$ for the category $H \mathcal{J}(S)\left(\mathbf{0}_{S},-\right) \downarrow \mathcal{S}^{H \mathcal{J}(S)}$ and call its objects pointed $H \mathcal{J}(S)$-spaces. (These are not $H \mathcal{J}(S)$-diagrams in pointed spaces!) The proof of Lemma 6.6 implies:

Corollary 6.8 The evaluation $\Gamma^{\mathrm{op}} \mathcal{J}-\mathcal{S} \rightarrow \mathcal{S}_{*}^{H \mathcal{J}(S)}$ preserves colimits. 
The functor $\alpha_{*}: H \mathcal{J}(S) \rightarrow H \mathcal{J}(T)$ induced by $\alpha: S \rightarrow T$ in $\Gamma^{\text {op }}$ gives rise to an adjunction

$$
\alpha_{!}: \mathcal{S}_{*}^{H \mathcal{J}(S)} \rightleftarrows \mathcal{S}_{*}^{H \mathcal{J}(T)}: \alpha^{*}
$$

The right adjoint is precomposition, and $\alpha_{*}\left(\mathbf{0}_{S}\right)=\mathbf{0}_{T}$ ensures that $\alpha^{*}(Y)\left(\mathbf{0}_{S}\right)$ is pointed. The left adjoint is defined by a left Kan extension.

Lemma 6.9 The evaluation functor $\Gamma^{\mathrm{op}} \mathcal{J}-\mathcal{S} \rightarrow \mathcal{S}_{*}^{H \mathcal{J}(S)}, X \mapsto X(S ;-)$ has a left adjoint $F_{S}$. For a pointed $H \mathcal{J}(S)$-space $Z$, there is an isomorphism

$$
\left(F_{S} Z\right)_{T} \cong \coprod_{\alpha: T} \alpha_{!}(Z),
$$

where $\alpha$ ranges over all non-zero maps in $\Gamma^{\mathrm{op}}$ and the coproduct is taken in $\mathcal{S}_{*}^{H \mathcal{J}(T)}$.

Proof We apply Lemma 6.4 to see that the right-hand side in (6-2) defines a $\Gamma^{\mathrm{op}} \mathcal{J}$ space. By (6-1) it is enough that there are maps $\hat{\beta}: \beta_{!}\left(F_{S} Z\right)_{T} \rightarrow\left(F_{S} Z\right)_{U}$ for every $\beta: T \rightarrow U$ in $\Gamma^{\mathrm{op}}$ such that $\widehat{\gamma}(\gamma ! \hat{\beta})=\widehat{\gamma \beta}$ holds for composable maps. On the summand indexed by $\alpha: S \rightarrow T$, we define $\hat{\beta}$ to be the inclusion of the summand $(\beta \alpha)_{!}(Z)$ if $\beta \alpha$ is non-zero. If $\beta \alpha$ factors as the composite $v \varepsilon$ with $v: 0^{+} \rightarrow U$, then $(\beta \alpha)_{!}(Z) \cong H \mathcal{J}(U)\left(0_{U},-\right) \times \varepsilon_{!}(Z)$, and we define $\beta_{!} \alpha_{!}(Z) \rightarrow\left(F_{S} Z\right)_{U}$ to be the map induced by the collapse of $\varepsilon !(Z)$ and the inclusion of the basepoint. It is easy to check that the $\widehat{\beta}$ satisfy the required compatibility and that this defines the desired left adjoint $F_{S}$.

Using various free/forgetful adjunctions, free $\Gamma^{\mathrm{op}} \mathcal{J}$-spaces on free pointed $H \mathcal{J}(S)$ spaces can also be expressed using the tensor introduced in Proposition 6.7:

Corollary 6.10 For objects $(s, \sigma)$ of $H \mathcal{J}(S)$, there is a natural isomorphism

$$
F_{S}\left(F_{\mathbf{0}_{S}}^{H \mathcal{J}(S)}(*) \amalg F_{(\boldsymbol{s}, \sigma)}^{H \mathcal{J}(S)}(K)\right) \cong \Gamma^{\mathrm{op}} \mathcal{J}((S ; \boldsymbol{s}, \sigma),-) \otimes K .
$$

\subsection{Commutative $\mathcal{J}$-space monoids and $\Gamma^{\mathrm{op}} \mathcal{J}$-spaces}

We give an alternative description of the passage from a commutative $\mathcal{J}$-space monoid to a $\Gamma^{\mathrm{op}} \mathcal{J}$-space provided by Corollary 6.5 in order to see that this construction is a right adjoint.

The free commutative $\mathcal{J}$-space monoid $\mathbb{C} F_{\left(\boldsymbol{n}_{1}, \boldsymbol{n}_{2}\right)}^{\mathcal{J}}(*)$ on a point in degree $\left(\boldsymbol{n}_{1}, \boldsymbol{n}_{2}\right)$ is the image of the free $\mathcal{J}$-space

$$
F_{\left(\boldsymbol{n}_{1}, \boldsymbol{n}_{2}\right)}^{\mathcal{J}}(*)=\mathcal{J}\left(\left(\boldsymbol{n}_{1}, \boldsymbol{n}_{2}\right),-\right)
$$


under the free functor $\mathbb{C}: \mathcal{S}^{\mathcal{J}} \rightarrow \mathcal{C S}^{\mathcal{J}}$ (compare (5-1)). It is contravariantly functorial in $\left(\boldsymbol{n}_{\mathbf{1}}, \boldsymbol{n}_{\mathbf{2}}\right)$, and using the coproduct in the category $\mathcal{C} \mathcal{S}^{\mathcal{J}}$ we obtain functors

$$
\mathbb{C} F_{S}: H \mathcal{J}(S)^{\mathrm{op}} \rightarrow \mathcal{C S}^{\mathcal{J}}, \quad(\boldsymbol{s}, \sigma) \mapsto \bigsqcup_{i \in \bar{S}} \mathbb{C F}_{\boldsymbol{s}_{i}}^{\mathcal{J}}(*) .
$$

Since the sum in $\mathcal{C S}^{\mathcal{J}}$ is the $\nabla$-product, a choice of an ordering of $\bar{S}$ induces an isomorphism between $\left(\mathbb{C} F_{S}\right)(\boldsymbol{s}, \sigma)$ and the iterated $\otimes$-product of the commutative $\mathcal{J}$-space monoids $\mathbb{C} F_{\boldsymbol{s}_{i}}^{\mathcal{J}}(*)$.

The functors $\mathbb{C} F_{S}$ for varying $S$ are related: a morphism $\alpha: S \rightarrow T$ in $\Gamma^{\text {op }}$ induces a natural transformation $\alpha^{*}: \mathbb{C F}_{T} \circ \alpha_{*} \rightarrow \mathbb{C F}_{S}$ of functors $H \mathcal{J}(S)^{\mathrm{op}} \rightarrow \mathcal{C} \mathcal{S}^{\mathcal{J}}$ such that, for a second morphism in $\beta: T \rightarrow U$ in $\Gamma^{\mathrm{op}}$, the composite

$$
\mathbb{C} F_{U} \circ \beta_{*} \circ \alpha_{*} \rightarrow \mathbb{C} F_{T} \circ \alpha_{*} \rightarrow \mathbb{C} F_{S}
$$

equals $(\beta \alpha)^{*}: \mathbb{C} F_{U} \circ(\beta \alpha)_{*} \rightarrow \mathbb{C} F_{S}$. The definition of $\alpha^{*}$ is similar to Section 3.9: choosing an ordering $\left\{i_{1}, \ldots, i_{v}\right\}$ of $V=\alpha^{-1}(j)$ for $j \in \bar{T}$, we have to define a map

$$
\mathbb{C} F_{\boldsymbol{s}_{V}}^{\mathcal{J}}(*) \rightarrow \mathbb{C} F_{\boldsymbol{s}_{i_{1}}}^{\mathcal{J}}(*) \otimes \cdots \otimes \mathbb{C} F_{\boldsymbol{s}_{i_{v}}}^{\mathcal{J}}(*) .
$$

It is equivalent to specify a point in the evaluation of the codomain at $s_{V}$. The canonical points in $\left(\mathbb{C} F_{\boldsymbol{s}_{i}}^{\mathcal{J}}(*)\right)\left(\boldsymbol{s}_{\boldsymbol{i}}\right)$ together with the morphism $s_{i_{1}} \sqcup \cdots \sqcup s_{i_{v}} \rightarrow s_{V}$ induced by the chosen ordering and the isomorphisms that are part of the data of $(s, \sigma)$ define such a point. The resulting map does not depend on the choices and satisfies the above naturality.

By the definition of the Grothendieck construction we obtain:

Corollary 6.12 The functors $\mathbb{C} F_{S}$ induce a functor $\mathbb{C} F:\left(\Gamma^{\mathrm{op}} \mathcal{J}\right)^{\mathrm{op}} \rightarrow \mathcal{C} \mathcal{S}^{\mathcal{J}}$ with $(\mathbb{C} F)(S ; s, \sigma)=(\mathbb{C} F)(s, \sigma)$.

The category of commutative $\mathcal{J}$-space monoids is tensored, cotensored and enriched over unpointed simplicial sets. Tensor and mapping space are defined by

$$
A \otimes K=\left|[m] \mapsto A^{\otimes K_{m}}\right| \quad \text { and } \quad \operatorname{Map}(A, B)=\left([m] \mapsto \mathcal{C S}^{\mathcal{J}}\left(A \otimes \Delta^{m}, B\right)\right),
$$

where $|-|$ denotes the realization of simplicial objects defined in terms of the diagonal. The cotensor is defined on the underlying $\mathcal{J}$-spaces. Together with this structure, the positive $\mathcal{J}$-model structure on $\mathcal{C S}^{\mathcal{J}}$ is a simplicial model category (in the sense of Hirschhorn [10, Definition 9.1.6]) since the positive $\mathcal{J}$-model structure on $\mathcal{J}$-space is simplicial [23, Proposition 6.19] and the compatibility with the model structure can be checked on the cotensor. 
Definition 6.13 For a commutative $\mathcal{J}$-space monoid $A$, we let $\Psi(A)$ be the $\Gamma^{\mathrm{op}} \mathcal{J}-$ space $\operatorname{Map}_{\mathcal{C S} \mathcal{J}}(\mathbb{C} F(-), A)$. For a $\Gamma^{\mathrm{op}} \mathcal{J}$-space $X$, we let $\Phi(X)$ be the coend over $\Gamma^{\text {op }} \mathcal{J}$ of the functor $\mathbb{C} F \otimes X$.

The adjunction of mapping space and tensor with a space imply:

Corollary 6.14 The functors $\Phi$ and $\Psi$ are the left and right adjoints in an adjunction $\Phi: \Gamma^{\mathrm{op}} \mathcal{J}-\mathcal{S} \rightleftarrows \mathcal{C S}^{\mathcal{J}}: \Psi$.

Since $\mathbb{C} F_{S}$ is defined as a coproduct of free commutative $\mathcal{J}$-space monoids, there is an isomorphism $\operatorname{Map}_{\mathcal{C S} \mathcal{J}}\left(\mathbb{C}_{S}^{\mathcal{J}}(*), A\right) \cong A_{S}$. Comparing the definition of the structure maps, we get an alternative description of the $\Gamma^{\mathrm{op}} \mathcal{J}$-space of Corollary 6.5:

Corollary 6.15 Let $A$ be a commutative $\mathcal{J}$-space monoid. The $\Gamma^{\mathrm{op}} \mathcal{J}$-space associated with the $H \mathcal{J}(S)$-spaces $A_{S}$ is isomorphic to $\Psi(A)$.

Generalizing the functor $\gamma: \mathcal{C} \mathcal{S}^{\mathcal{J}} \rightarrow \Gamma^{\mathrm{op}}-\mathcal{S}$ introduced in Definition 3.11, we obtain a functor $\gamma^{\prime}: \Gamma^{\mathrm{op}} \mathcal{J}-\mathcal{S} \rightarrow \Gamma^{\mathrm{op}}-\mathcal{S}$ by setting $\gamma^{\prime}(X)(S)=X(S ;-)_{h H \mathcal{J}(S)}$.

Corollary 6.16 There is a natural isomorphism $\gamma^{\prime}(\Psi(A)) \cong \gamma(A)$.

Remark 6.17 The usual bar construction $B A=B(*, A, *)$ in $\mathcal{C S}^{\mathcal{J}}$ seems to be of limited use for our purposes since the terminal object of $\mathcal{C} \mathcal{S}^{\mathcal{J}}$ is not initial. (This means in particular that we cannot form $\Omega(B A)$.)

However, one may interpret $\Psi$ as a generalized bar construction: $\Psi(A)$ encodes iterated products of the spaces $A\left(\boldsymbol{m}_{\mathbf{1}}, \boldsymbol{m}_{\mathbf{2}}\right)$ and the structure maps between them induced by the multiplication and unit of $A$, without ever attempting to realize this as an object of $\mathcal{C S}^{\mathcal{J}}$. Corollary 6.14 justifies why the more elaborate category $\Gamma^{\text {op }} \mathcal{J}-\mathcal{S}$ is an appropriate codomain of such a generalized bar construction.

\subsection{Level model structures on $\Gamma^{\mathrm{op}} \mathcal{J}$-spaces}

Standard model category arguments (see eg Hirschhorn [10, Section 11.6]) show that the category $\Gamma^{\mathrm{op}}-\mathcal{S}$ admits a cofibrantly generated proper level model structure in which a map $X \rightarrow Y$ is a weak equivalence or a fibration if and only if $X(S) \rightarrow Y(S)$ is a weak equivalence or fibration of spaces for every finite based set $S$. The cofibrations are determined by the left lifting property. Following Schwede's terminology [27], we call this the level $Q$-model structure and write $\left(\Gamma^{\mathrm{op}}-\mathcal{S}\right)_{\text {lev }}$ for this model category. It is the first step towards the stable Q-model structure on $\Gamma^{\mathrm{op}}-\mathcal{S}$ appearing in Theorem 5.10.

Next we will build a corresponding "level" model structure on the category $\Gamma^{\mathrm{op}} \mathcal{J}-\mathcal{S}$. It is based on model structures on the categories $\mathcal{S}^{H \mathcal{J}(S)}$ we will treat first. 
Definition 6.19 An object $(s, \sigma)$ in $H \mathcal{J}(S)$ is positive if for every $i \in \bar{S}$ the object $\boldsymbol{s}_{i}=\left(\boldsymbol{m}_{\mathbf{1}}, \boldsymbol{m}_{\mathbf{2}}\right)$ of $\mathcal{J}$ satisfies $m_{1} \geq 1$.

A map $X \rightarrow Y$ in $\mathcal{S}^{H \mathcal{J}(S)}$ is a $H \mathcal{J}(S)$-equivalence if the induced map of homotopy colimits hocolim $\operatorname{HJJ}_{(S)} X \rightarrow \operatorname{hocolim}_{H \mathcal{J}(S)} Y$ is a weak equivalence of spaces. It is a positive $H \mathcal{J}(S)$-fibration if for every morphism $f:(\boldsymbol{s}, \sigma) \rightarrow(\boldsymbol{t}, \tau)$ in $H \mathcal{J}(S)$ between positive objects the induced square

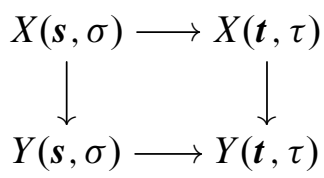

is a homotopy cartesian square in which the vertical maps are Kan fibrations. Positive $H \mathcal{J}(S)$-cofibrations are the maps with the left lifting property with respect to all maps which are both positive $H \mathcal{J}(S)$-fibrations and $H \mathcal{J}(S)$-equivalences.

A map $X \rightarrow Y$ in $\mathcal{S}^{H \mathcal{J}(S)}$ is an absolute $H \mathcal{J}(S)$-fibration if it satisfies the condition of a positive $H \mathcal{J}(S)$-fibration for all morphisms of $H \mathcal{J}(S)$ (without requiring that their domains and codomains are positive). The absolute $H \mathcal{J}(S)$-cofibrations are the maps with the left lifting property with respect to all maps which are both absolute $H \mathcal{J}(S)$-fibrations and $H \mathcal{J}(S)$-equivalences.

Lemma 6.20 If $S$ is non-trivial, the $H \mathcal{J}(S)$-equivalences, the positive $H \mathcal{J}(S)$ fibrations and the positive $H \mathcal{J}(S)$-cofibrations define a cofibrantly generated proper simplicial positive $H \mathcal{J}(S)$-model structure. The same holds in the absolute case.

We write $\mathcal{S}_{\text {pos }}^{H \mathcal{J}(S)}$ and $\mathcal{S}_{\text {abs }}^{H \mathcal{J}(S)}$ for these model categories. It is immediate that the identity functors form a Quillen equivalence $\mathcal{S}_{\text {pos }}^{H \mathcal{J}(S)} \rightleftarrows \mathcal{S}_{\text {abs }}^{H \mathcal{J}(S)}$.

Proof We apply the general existence result for such "homotopy colimit model structures" on diagram categories [23, Proposition 6.16]. Using this, it remains to show that $H \mathcal{J}(S)$ together with the discrete subcategory on the identity morphisms of positive objects (resp. all objects in the absolute case) is a well-structured relative index category in the sense of [23, Definition 5.2]. By [23, Corollary 5.9], this is the case for $\mathcal{J}$. If one defines the degree function of a product to be the sum of the degree functions of the factors, this structure is preserved under products. It is also preserved under equivalences of symmetric monoidal categories. Hence Lemma 3.4 shows the claim for $H \mathcal{J}(S)$. Properness is shown in [23, Section 11]. By [23, Proposition 6.19], the model structure is simplicial. 
Remark 6.21 We use a positive model structure because of the connection to commutative $\mathcal{J}$-space monoids in Lemma 6.26 below. The absolute counterpart is needed in some proofs since it has the advantage that the map $\alpha^{*}: \mathcal{S}_{\mathrm{abs}}^{H \mathcal{J}(T)} \rightarrow \mathcal{S}_{\mathrm{abs}}^{H \mathcal{J}(S)}$ induced by $\alpha: S \rightarrow T$ preserves fibrations and weak equivalences. This is does not hold in the positive case because $\alpha^{*}(X)$ may evaluate $X$ at $\boldsymbol{s}_{\varnothing}=(\mathbf{0}, \mathbf{0})$ in some components if $\alpha$ is not surjective.

We now use these model categories for varying $S$ to construct model structures on the category of $\Gamma^{\mathrm{op}} \mathcal{J}$-spaces. A map $f: X \rightarrow Y$ in $\Gamma^{\mathrm{op}} \mathcal{J}-\mathcal{S}$ is a level HJ-equivalence if the map $X(S ;-) \rightarrow Y(S ;-)$ is an $H \mathcal{J}(S)$-equivalence for every finite based set $S$. It is a positive level $H \mathcal{J}$-fibration if $X(S ;-) \rightarrow Y(S ;-)$ is a positive $H \mathcal{J}(S)$-fibration for every finite based set $S$. The positive level $H \mathcal{J}$-cofibrations are the maps with the left lifting property with respect to all maps which are both level $H \mathcal{J}$-equivalences and positive level $H \mathcal{J}$-fibrations.

Proposition 6.22 The level $H \mathcal{J}$-equivalences, the positive level $H \mathcal{J}$-fibrations and the positive level $H \mathcal{J}$-cofibrations define a cofibrantly generated simplicial positive level model structure on the category $\Gamma^{\mathrm{op}} \mathcal{J}-\mathcal{S}$.

Proof The adjunction of Lemma 6.9 induces an adjunction

$$
F: \prod_{S \neq 0^{+}} \mathcal{S}_{*}^{H \mathcal{J}(S)} \rightleftarrows \Gamma^{\mathrm{op}} \mathcal{J}-\mathcal{S}: U
$$

in which $U$ is the evident forgetful functor and $F\left(\left(Z_{S}\right)_{S}\right)=\bigsqcup F_{S}\left(Z_{S}\right)$. For every $S$, the comma category

$$
\mathcal{S}_{*}^{H \mathcal{J}(S)}=H \mathcal{J}(S)\left(\mathbf{0}_{S},-\right) \downarrow \mathcal{S}^{H \mathcal{J}(S)}
$$

inherits a cofibrantly generated positive $H \mathcal{J}(S)$-model structure from $\mathcal{S}^{H \mathcal{J}(S)}$. We apply the general lifting criterion of a cofibrantly generated model structure along a right adjoint provided by [10, Theorem 11.3.2]. Since each of the $\mathcal{S}_{*}^{H \mathcal{J}(S)}$ is cofibrantly generated, so is their product [10, Proposition 11.1.10]. The category $\Gamma^{\mathrm{op}} \mathcal{J}-\mathcal{S}$ is locally presentable, so all objects are small relative to the whole category.

It remains to show that for a set of generating acyclic cofibrations $J$ of the product model structure, $U$ sends relative $F J$-cell complexes to weak equivalences. For this we use that the $\left(\alpha_{!}, \alpha^{*}\right)$ are Quillen adjunctions for the absolute model structures. The explicit description of $F_{S}$ in Lemma 6.9 shows that each component of a relative cell complex built from maps in $F(J)$ is an absolute acyclic $H \mathcal{J}(S)$-cofibration. Since the positive model structures have less acyclic cofibrations and the same weak equivalences than the absolute ones, this implies the claim. 
The property of being simplicial is inherited from the $H \mathcal{J}(S)$-model structures because it is enough to check this condition on the cotensors.

We write $\left(\Gamma^{\mathrm{op}} \mathcal{J}-\mathcal{S}\right)_{\text {lev }}$ for the model category resulting from Proposition 6.22.

Remark 6.23 We call this the level model structure because it is related to $\left(\Gamma^{\mathrm{op}}-\mathcal{S}\right)_{\text {lev }}$ by Proposition 6.29 below. There are also level model structures on $H \mathcal{J}(S)$-spaces, but those will not be considered in this paper.

The argument used in the proof of the last proposition also implies:

Corollary 6.24 The positive level $H \mathcal{J}$-cofibrations are levelwise absolute $H \mathcal{J}(S)$ cofibrations.

Corollary 6.25 The positive level $H \mathcal{J}$-model structure is proper.

Proof Right properness lifts from the positive $H \mathcal{J}(S)$-model structure. With the previous corollary, left properness of the absolute $H \mathcal{J}(S)$-model structure implies left properness of the positive level $H \mathcal{J}$-model structure.

Lemma 6.26 The adjunction $\Phi:\left(\Gamma^{\mathrm{op}} \mathcal{J}-\mathcal{S}\right)_{\text {lev }} \rightleftarrows \mathcal{C S}^{\mathcal{J}}: \Psi$ of Corollary 6.14 is a Quillen adjunction with respect to the positive $\mathcal{J}$-model structure on $\mathcal{C} \mathcal{S}^{\mathcal{J}}$.

Proof The identification of the right adjoint in Corollary 6.15 makes it easy to check that it preserves weak equivalences and fibrations.

\subsection{7 $\Gamma^{\mathrm{op}} \mathcal{J}$-spaces and augmented $\Gamma$-spaces}

We will now relate $\Gamma^{\mathrm{op}} \mathcal{J}$-spaces to ordinary $\Gamma$-spaces by applying the comparison between $\mathcal{K}$-spaces and spaces over $B \mathcal{K}$ of [23, Theorem 13.2] levelwise.

As in Definition 2.2, let $\mathcal{S}^{\mathcal{K}}$ be the category of $\mathcal{K}$-spaces for a small category $\mathcal{K}$. Viewing the comma category $(\mathcal{K} \downarrow \boldsymbol{k})$ as a functor in $\boldsymbol{k}$, we obtain a $\mathcal{K}$-space $E \mathcal{K}=B(\mathcal{K} \downarrow-)$. Composition with $E \mathcal{K} \rightarrow *$ and the product with $E \mathcal{K}$ provide the first adjunction in

$$
\mathcal{S}^{\mathcal{K}} \leftrightarrows \mathcal{S}^{\mathcal{K}} / E \mathcal{K} \rightleftarrows \mathcal{S} / B \mathcal{K}
$$

The right adjoint in the second adjunction is obtained by viewing a map of spaces $Y \rightarrow B \mathcal{K}$ as a map of constant $\mathcal{K}$-spaces and forming the pullback $E \mathcal{K} \times{ }_{B \mathcal{K}} Y$ along the map $E \mathcal{K} \rightarrow B \mathcal{K}$ induced by the projection $(\mathcal{K} \downarrow \boldsymbol{k}) \rightarrow \mathcal{K}$. Its left adjoint is obtained by applying $\operatorname{colim}_{\mathcal{K}}$ and composing with $\operatorname{colim}_{\mathcal{K}} E \mathcal{K} \cong B \mathcal{K}$. 
Lemma 6.28 Let $S$ be a non-trivial finite based set. Then for $\mathcal{K}=H \mathcal{J}(S)$, the diagram (6-4) is a chain of Quillen equivalences with respect to the positive $H \mathcal{J}(S)$-model structure on $\mathcal{S}^{H \mathcal{J}(S)}$, the positive overcategory model structure on $\mathcal{S}^{H \mathcal{J}(S)} / E H \mathcal{J}(S)$ and the overcategory model structure on $\mathcal{S} / B H \mathcal{J}(S)$. The same holds in the absolute case.

Proof We used in the proof of Lemma 6.20 that $H \mathcal{J}(S)$ is a well-structured index category in the sense of [23, Definition 5.5]. Hence [23, Theorem 13.2] applies and provides the desired Quillen equivalences for the absolute $H \mathcal{J}(S)$-model structures on $H \mathcal{J}(S)$-spaces. Since the positive model structures have the same weak equivalences and less cofibrations than the absolute ones, this implies the claim.

The $\mathcal{K}$-space $E \mathcal{K}$ appearing in (6-4) is functorial in $\mathcal{K}:$ a functor $F: \mathcal{K} \rightarrow \mathcal{L}$ induces a natural transformation $E \mathcal{K} \rightarrow F^{*}(E \mathcal{L})$ of $\mathcal{K}$-spaces. Inspecting the definition of the Grothendieck construction, one verifies that these natural transformations make the collection of $H \mathcal{J}(S)$-spaces $E H \mathcal{J}(S)$ into a $\Gamma^{\mathrm{op}} \mathcal{J}$-space $E H \mathcal{J}$.

Proposition 6.29 There is a chain of Quillen equivalences

$$
\left(\Gamma^{\mathrm{op}} \mathcal{J}-\mathcal{S}\right)_{\mathrm{lev}} \leftrightarrows\left(\Gamma^{\mathrm{op}} \mathcal{J}-\mathcal{S}\right)_{\mathrm{lev}} / E H \mathcal{J} \rightleftarrows\left(\Gamma^{\mathrm{op}}-\mathcal{S}\right)_{\mathrm{lev}} / b \mathcal{J}
$$

induced by the chain of adjunctions (6-4).

Proof The first adjunction is induced by the level $H \mathcal{J}$-equivalence $E H \mathcal{J} \rightarrow *$. It is a Quillen equivalence since $\left(\Gamma^{\mathrm{op}} \mathcal{J}-\mathcal{S}\right)_{\text {lev }}$ is right proper.

Using the description of the category $\Gamma^{\mathrm{op}} \mathcal{J}-\mathcal{S}$ in Lemma 6.4 one can check that the adjunctions $\mathcal{S}^{H \mathcal{J}(S)} / E H \mathcal{J}(S) \rightleftarrows \mathcal{S} / B H \mathcal{J}(S)$ defined in (6-4) and the maps $E H \mathcal{J}(S) \rightarrow \alpha^{*}(E H \mathcal{J}(T))$ do indeed induce the second adjunction in (6-5). The positive instance of the Quillen equivalence $\mathcal{S}^{H \mathcal{J}(S)} / E H \mathcal{J}(S) \rightleftarrows \mathcal{S} / B H \mathcal{J}(S)$ of Lemma 6.28 and the definition of the model structures imply that the right adjoint is a right Quillen functor. Corollary 6.24 and the absolute case of Lemma 6.28 show that it is a Quillen equivalence.

We write $\mathcal{K}$ for $H \mathcal{J}(S)$ for the rest of this section. The composed derived functor of the Quillen equivalences (6-4) sends a $\mathcal{K}$-space $Z$ to $\operatorname{colim}_{\mathcal{K}}\left(\left(Z^{\text {fib }} \times E \mathcal{K}\right)^{\text {cof }}\right)$. (Here composed derived functor has the same meaning as in Theorem 5.10.)

The maps $\left(Z^{\mathrm{fib}} \times E \mathcal{K}\right)^{\mathrm{cof}} \rightarrow Z^{\mathrm{fib}} \times E \mathcal{K} \rightarrow Z^{\mathrm{fib}} \leftarrow Z$ and the natural map from the homotopy colimit to the colimit induce weak equivalences

$$
Z_{h \mathcal{K}} \rightarrow\left(Z^{\mathrm{fib}}\right)_{h \mathcal{K}} \leftarrow\left(\left(Z^{\mathrm{fib}} \times E \mathcal{K}\right)^{\mathrm{cof}}\right)_{h \mathcal{K}} \rightarrow \operatorname{colim}_{\mathcal{K}}\left(\left(Z^{\mathrm{fib}} \times E \mathcal{K}\right)^{\mathrm{cof}}\right)
$$

by [23, Lemma 6.22] and the right properness of the $H \mathcal{J}(S)$-model structure. 
Corollary 6.30 After forgetting the augmentation to $B H \mathcal{J}(S)$, the value of the composed derived functor of the chain of Quillen equivalences of Lemma 6.28 at an $H \mathcal{J}(S)$-space $Z$ is weakly equivalent to the homotopy colimit $Z_{h H \mathcal{J}(S)}$.

To simplify the notation we now assume that $Z$ is positive $H \mathcal{J}(S)$-fibrant and compare $Z_{h \mathcal{K}}$ and $\operatorname{colim}_{\mathcal{K}}\left((Z \times E \mathcal{K})^{\text {cof }}\right)$ as spaces over $B \mathcal{K}$. This is more subtle since $Z \rightarrow *$ induces the augmentation $Z_{h \mathcal{K}} \rightarrow(*)_{h \mathcal{K}} \cong B \mathcal{K}$ of the former, while the augmentation of latter is induced by the maps $(Z \times E \mathcal{K})^{\text {cof }} \rightarrow E \mathcal{K}$ and $\operatorname{colim}_{\mathcal{K}} E \mathcal{K} \cong B \mathcal{K}$. These maps fit into a commutative diagram

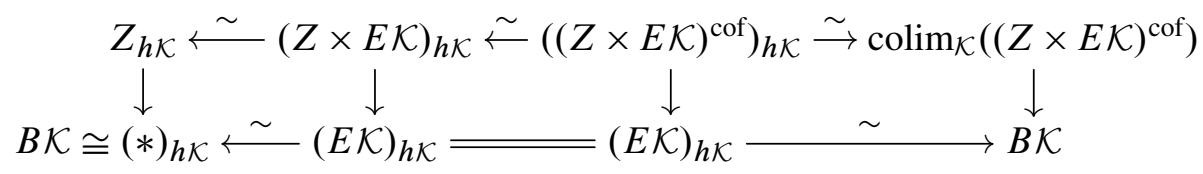

in which the two maps $(E \mathcal{K})_{h \mathcal{K}} \rightarrow B \mathcal{K}$ in the bottom line differ. So the zig-zag of weak equivalences (6-6) does not lie over $B \mathcal{K}$. However, using the notation $(*)_{h \mathcal{K}}$ to distinguish the two copies of $B \mathcal{K}$, we have:

Lemma 6.31 Composition with and base change along the bottom maps in (6-7) induce a chain of Quillen equivalences

$$
\mathcal{S} /(*)_{h H \mathcal{J}(S)} \leftrightarrows \mathcal{S} /(E H \mathcal{J}(S))_{h H \mathcal{J}(S)} \rightleftarrows \mathcal{S} / B H \mathcal{J}(S)
$$

For an $H \mathcal{J}(S)$-space $Z$, there is a chain of $H \mathcal{J}(S)$-equivalences relating the value of $Z_{h H \mathcal{J}(S)} \rightarrow(*)_{h H \mathcal{J}(S)}$ under the composed derived functor of this chain of Quillen equivalences with $\operatorname{colim}_{\mathcal{K}}\left(\left(Z^{\text {fib }} \times E \mathcal{K}\right)^{\text {cof }}\right) \rightarrow B H \mathcal{J}(S)$.

Proof Right properness of $\mathcal{S}$ implies that composition with and pullback along a weak equivalence induces a Quillen equivalence of comma categories. By [23, Corollary 11.4], the left-hand square in (6-7) is homotopy cartesian. Hence (6-7) computes the composed derived functor and provides the desired chain of equivalences.

\section{Stable model structures on $\Gamma^{\text {op }} \mathcal{J}$-spaces}

In this section we build pre-stable and stable model structures on the category of $\Gamma^{\mathrm{op}} \mathcal{J}$-spaces and use them to prove Theorem 5.5 and Theorem 5.10. 


\subsection{Left Bousfield localizations}

Below we will frequently employ left Bousfield localizations and therefore recall some terminology about these. To simplify this, we only consider localizations of simplicial model categories. We refer to Hirschhorn's book [10] as an extensive reference about left Bousfield localizations.

Let $\mathcal{M}$ be left proper simplicial model category and $S$ be a set of maps between cofibrant objects in $\mathcal{M}$. An object $W$ of $\mathcal{M}$ is called $S$-local if it is fibrant in $\mathcal{M}$ and if every $f: X \rightarrow Y$ in $S$ induces a weak equivalence $f^{*}: \operatorname{Map}(Y, W) \rightarrow \operatorname{Map}(X, W)$ of simplicial mapping spaces. A map of cofibrant objects $g: X \rightarrow Y$ is an $S$-local equivalence if the induced map $g^{*}: \operatorname{Map}(Y, W) \rightarrow \operatorname{Map}(X, W)$ of simplicial mapping spaces is a weak equivalence for every $S$-local object $W$. A general map $g$ is an $S$-local equivalence if the induced map of cofibrant replacements is.

If it exists, the left Bousfield localization of $\mathcal{M}$ with respect to $S$ is a new model category structure on $\mathcal{M}$ with the same cofibrations as before and the $S$-local equivalences as weak equivalences. The fibrations are determined by a lifting property. Left properness of $\mathcal{M}$ ensures that the fibrant objects of the localization are the $S$-local objects [10, Proposition 3.4.1]. Left Bousfield localizations exist if $\mathcal{M}$ is sufficiently well-behaved, for example left proper and cellular [10, Theorem 4.1.1] or left proper and combinatorial; see Barwick [4, Theorem 4.7].

\subsection{Pre-stable model structures}

Lemma 6.26 and Proposition 6.29 provide a chain of one Quillen adjunction and two Quillen equivalences

$$
\mathcal{C S}^{\mathcal{J}} \leftrightarrows\left(\Gamma^{\mathrm{op}} \mathcal{J}-\mathcal{S}\right)_{\text {lev }} \leftrightarrows\left(\Gamma^{\mathrm{op}} \mathcal{J}-\mathcal{S}\right)_{\text {lev }} / E H \mathcal{J} \rightleftarrows\left(\Gamma^{\mathrm{op}}-\mathcal{S}\right)_{\text {lev }} / b \mathcal{J}
$$

with respect to the positive $\mathcal{J}$-model on $\mathcal{C S}^{\mathcal{J}}$ and the (positive) level model structures on the remaining categories. We will now localize the three model categories on the right-hand side so that all adjunctions in (7-1) become Quillen equivalences. The idea is to build certain pre-stable model structures that lie between the level model structures of the last paragraph and the stable model structures that occur in the identification of the group completion model structure in Theorem 5.10.

We begin with ordinary $\Gamma$-spaces. Let $p_{S}: S \vee T \rightarrow S$ and $p_{T}: S \vee T \rightarrow T$ be the projections associated with a pair of objects in $\Gamma^{\mathrm{op}}$. We consider the set of maps

$$
P^{\prime}=\left\{p_{S}^{*} \vee p_{T}^{*}: \Gamma^{\mathrm{op}}(S,-) \vee \Gamma^{\mathrm{op}}(T,-) \rightarrow \Gamma^{\mathrm{op}}(S \vee T,-) \mid S, T \in \mathrm{Ob}\left(\Gamma^{\mathrm{op}}\right)\right\}
$$

in $\Gamma^{\mathrm{op}}-\mathcal{S}$. 
Lemma 7.3 The left Bousfield localization of $\left(\Gamma^{\mathrm{op}}-\mathcal{S}\right)_{\text {lev }}$ with respect to $P^{\prime}$ exists. It is a cofibrantly generated left proper model category.

Proof The category $\Gamma^{\mathrm{op}}-\mathcal{S}$ is locally presentable, and $\left(\Gamma^{\mathrm{op}}-\mathcal{S}\right)_{\text {lev }}$ is cofibrantly generated and left proper. Hence Smith's existence theorem for left Bousfield localizations of combinatorial model categories applies; see Barwick [4, Theorem 4.7].

We write $\left(\Gamma^{\mathrm{op}}-\mathcal{S}\right)_{\text {pre }}$ for the resulting pre-stable model structure on $\Gamma^{\mathrm{op}}-\mathcal{S}$ and call its weak equivalences pre-stable equivalences. The above characterization of fibrant objects in the localization implies that its fibrant objects are the levelwise fibrant $\Gamma$-spaces which are special (as defined in Section 3.1).

Remark 7.4 The pre-stable model structure is related to Segal's original definition of $\Gamma$-spaces [29]. In our terminology, Segal only considers special $\Gamma$-spaces, and the homotopy category of the model category $\left(\Gamma^{\mathrm{op}}-\mathcal{S}\right)_{\text {pre }}$ is a model for the homotopy category of special $\Gamma$-spaces that Segal uses. The homotopy category of special $\Gamma$-spaces is also considered by Mandell [13] who proves that it is equivalent to the homotopy categories of $E_{\infty}$ spaces and of permutative categories.

To obtain pre-stable model structures on $\Gamma^{\mathrm{op}} \mathcal{J}$-spaces, we need to choose a set of maps similar to $P^{\prime}$. If $(\boldsymbol{u}, v)$ is an object of $H \mathcal{J}(S \vee T)$, the projections $p_{S}$ and $p_{T}$ induce morphisms $p_{S}=\left(p_{S} ;\right.$ id $):(S \vee T ; \boldsymbol{u}, v) \rightarrow\left(S ;\left(p_{S}\right)_{*}(\boldsymbol{u}, v)\right)$ and $p_{T}=\left(p_{T} ;\right.$ id $)$ in $\Gamma^{\mathrm{op}} \mathcal{J}$. These maps in turn induce morphisms

$$
\begin{gathered}
\Gamma^{\mathrm{op}} \mathcal{J}\left(\left(S ;\left(p_{S}\right)_{*}(\boldsymbol{u}, v)\right),-\right) \amalg \Gamma^{\mathrm{op}} \mathcal{J}\left(\left(T ;\left(p_{T}\right)_{*}(\boldsymbol{u}, v)\right),-\right) \\
\downarrow p_{S}^{*} \vee p_{T}^{*} \\
\Gamma^{\mathrm{op}} \mathcal{J}((S \vee T ; \boldsymbol{u}, v),-)
\end{gathered}
$$

of the free $\Gamma^{\mathrm{op}} \mathcal{J}$-spaces represented by the objects involved.

Definition 7.5 Let $P$ be the set of maps in $\Gamma^{\text {op }} \mathcal{J}-\mathcal{S}$ of the form (7-2) where $S$ and $T$ are non-trivial objects of $\Gamma^{\mathrm{op}}$ and $(\boldsymbol{u}, v)$ is a positive object of $H \mathcal{J}(S \vee T)$. Let $P_{E}$ be the set of all maps in $\left(\Gamma^{\mathrm{op}} \mathcal{J}-\mathcal{S}\right) / E H \mathcal{J}$ whose projection to $\Gamma^{\mathrm{op}} \mathcal{J}-\mathcal{S}$ is a map in $P$, and let $P_{B}$ be the image of $P_{E}$ under the left adjoint $\left(\Gamma^{\mathrm{op}} \mathcal{J}-\mathcal{S}\right) / E H \mathcal{J} \rightarrow\left(\Gamma^{\mathrm{op}}-\mathcal{S}\right) / b \mathcal{J}$.

Lemma 7.6 The image of $P_{E}$ under $\Gamma^{\mathrm{op}} \mathcal{J}-\mathcal{S} / E H \mathcal{J} \rightarrow \Gamma^{\mathrm{op}} \mathcal{J}-\mathcal{S}$ is $P$.

Proof It is enough to show that there exists a map $\Gamma^{\mathrm{op}} \mathcal{J}((S \vee T ; \boldsymbol{u}, v),-) \rightarrow E H \mathcal{J}$ in $\Gamma^{\mathrm{op}} \mathcal{J}-\mathcal{S}$. This holds because the space $E H \mathcal{J}(S \vee T ; \boldsymbol{u}, v) \cong B(H \mathcal{J}(S \vee T) \downarrow(\boldsymbol{u}, v))$ is non-empty. 
Lemma 7.7 The left Bousfield localizations of $\left(\Gamma^{\mathrm{op}} \mathcal{J}-\mathcal{S}\right)_{\text {lev }}$ with respect to $P$, of $\left(\Gamma^{\mathrm{op}} \mathcal{J}-\mathcal{S}\right)_{\text {lev }} / E H \mathcal{J}$ with respect to $P_{E}$, and of $\left(\Gamma^{\mathrm{op}}-\mathcal{S}\right)_{\text {lev }} / b \mathcal{J}$ with respect to $P_{B}$ exist. They are cofibrantly generated and left proper.

Proof Since the categories are locally presentable and the level model structures are cofibrantly generated and left proper, Smith's existence theorem for left Bousfield localizations [4, Theorem 4.7] applies.

We refer to these model structures as the pre-stable model structures, write $\left(\Gamma^{\mathrm{op}} \mathcal{J}-\mathcal{S}\right)_{\text {pre }}$, $\left(\left(\Gamma^{\mathrm{op}} \mathcal{J}-\mathcal{S}\right) / E H \mathcal{J}\right)_{\text {pre }}$, and $\left(\left(\Gamma^{\mathrm{op}}-\mathcal{S}\right) / b \mathcal{J}\right)_{\text {pre }}$ for these model categories and call the weak equivalences pre-stable equivalences.

It is important to note (and non-trivial to prove) that two possibly distinct model structures on $\Gamma^{\mathrm{op}}-\mathcal{S} / b \mathcal{J}$ arising from these constructions coincide:

Lemma 7.8 The overcategory model structure $\left(\Gamma^{\mathrm{op}}-\mathcal{S}\right)_{\text {pre }} / b \mathcal{J}$ and the pre-stable model structure $\left(\Gamma^{\mathrm{op}}-\mathcal{S} / b \mathcal{J}\right)_{\text {pre }}$ of the last lemma coincide.

Proof Let $P_{B}^{\prime}$ be the set of all maps in $\Gamma^{\mathrm{op}}-\mathcal{S} / b \mathcal{J}$ whose projection to $\Gamma^{\mathrm{op}}-\mathcal{S}$ is in $P^{\prime}$. Since the levelwise colimit of a free $\Gamma^{\mathrm{op}} \mathcal{J}$-space is a free $\Gamma$-space, the left adjoint $\Gamma^{\mathrm{op}} \mathcal{J}-\mathcal{S} / E H \mathcal{J} \rightarrow \Gamma^{\mathrm{op}}-\mathcal{S} / b \mathcal{J}$ sends maps in $P_{E}$ to maps in $P_{B}^{\prime}$. We claim that up to isomorphism every map in $P_{B}^{\prime}$ is in the image. For this we have to show that every possible augmentation $\Gamma^{\mathrm{op}}(S,-) \rightarrow b \mathcal{J}$ of the codomain of a map in $P^{\prime}$ is in the image. Such maps correspond to objects $(s, \sigma)$ of $H \mathcal{J}(S)$, and the map $\left.\Gamma^{\mathrm{op}} \mathcal{J}(S ; \boldsymbol{s}, \sigma),-\right) \rightarrow E H \mathcal{J}$ specified by the point in $B(H \mathcal{J}(S) \downarrow(s, \sigma))$ given by the identity on $(s, \sigma)$ is the desired preimage under the left adjoint. This proves the claim about $P_{B}^{\prime}$.

It remains to prove that the localization of $\Gamma^{\mathrm{op}}-\mathcal{S} / b \mathcal{J}$ with respect to $P_{B}^{\prime}$ is the overcategory model structure $\left(\Gamma^{\mathrm{o}} \mathrm{p}_{-} \mathcal{S}\right)_{\text {pre }} / b \mathcal{J}$. Since all maps in $P_{B}^{\prime}$ are weak equivalences in $\left(\Gamma^{\mathrm{op}}-\mathcal{S}\right)_{\text {pre }}$ when forgetting the augmentation, the identity functor is a left Quillen functor $\left(\Gamma^{\mathrm{op}}-\mathcal{S} / b \mathcal{J}\right)_{\text {pre }} \rightarrow\left(\Gamma^{\mathrm{op}}-\mathcal{S}\right)_{\text {pre }} / b \mathcal{J}$. To see that the model structures coincide, it is therefore enough to show that the fibrant objects in $\left(\Gamma^{\mathrm{op}}-\mathcal{S} / b \mathcal{J}\right)_{\text {pre }}$ are fibrant in $\left(\Gamma^{\mathrm{op}}-\mathcal{S}\right)_{\text {pre }} / b \mathcal{J}$. An object $f: X \rightarrow b \mathcal{J}$ is fibrant in $\left(\Gamma^{\mathrm{op}}-\mathcal{S} / b \mathcal{J}\right)_{\text {pre }}$ if $f$ is a level fibration in $\Gamma^{\mathrm{o}} \mathrm{P}_{-} \mathcal{S}$ and every map $g: U \rightarrow V$ in $P_{B}^{\prime}$ induces a weak equivalence $\operatorname{Map}_{b \mathcal{J}}(g, X)$. The space $\operatorname{Map}_{b \mathcal{J}}(U, X)$ of maps from $U \rightarrow b \mathcal{J}$ to $f$ in $\Gamma^{\mathrm{op}}-\mathcal{S} / b \mathcal{J}$ can be defined as the fiber of $f_{*}: \operatorname{Map}_{\Gamma_{\mathrm{op}_{-} \mathcal{S}}}(U, X) \rightarrow \operatorname{Map}_{\Gamma^{\text {op }} \mathcal{S}}(U, b \mathcal{J})$ over the augmentation $U \rightarrow b \mathcal{J}$ of $U$. Since $f$ is a level fibration, $f_{*}$ is a fibration 
of spaces. Hence every $g: U \rightarrow V$ in $P_{B}^{\prime}$ induces a map of homotopy fiber sequences

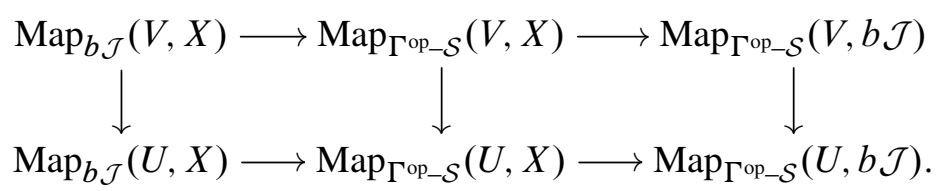

By assumption, the first and third vertical maps are weak equivalences. Since the maps $g$ in $P_{B}^{\prime}$ that project to a given map $U \rightarrow V$ in $P^{\prime}$ vary over all possible maps over $b \mathcal{J}$, we obtain the previous diagram of homotopy fiber sequences for all possible basepoints in $\operatorname{Map}_{\Gamma^{\text {op_-S }}}(V, b \mathcal{J})$. It follows that the middle vertical map is a weak equivalence for all $U \rightarrow V$ in $P^{\prime}$. Let $f^{\prime}: X^{\prime} \rightarrow b \mathcal{J}^{\prime}$ be a level fibration of level fibrant objects that is level equivalent to $f$. Since the $U$ is a coproduct of free $\Gamma$-spaces, the mapping space $\operatorname{Map}_{\Gamma^{\text {op }} \mathcal{S}}(U,-)$ maps level equivalences between all objects to weak equivalences, and it follows that $X^{\prime}$ and $b \mathcal{J}^{\prime}$ are fibrant in $\left(\Gamma^{\mathrm{op}}-\mathcal{S}\right)_{\text {pre }}$. So $f^{\prime}$ is a fibration in $\left(\Gamma^{\mathrm{op}}-\mathcal{S}\right)_{\text {pre }}$ by [10, Proposition 3.4.7]. Forming the base change along $b \mathcal{J} \rightarrow b \mathcal{J}^{\prime}$, [10, Proposition 3.4.6] shows that $f$ is a fibration in $\left(\Gamma^{\mathrm{op}}-\mathcal{S}\right)_{\text {pre }}$.

Corollary 7.9 There is a chain of Quillen equivalences

$$
\left(\Gamma^{\mathrm{op}} \mathcal{J}-\mathcal{S}\right)_{\mathrm{pre}} \leftrightarrows\left(\Gamma^{\mathrm{op}} \mathcal{J}-\mathcal{S}\right)_{\mathrm{pre}} / E H \mathcal{J} \rightleftarrows\left(\Gamma^{\mathrm{op}}-\mathcal{S}\right)_{\mathrm{pre}} / b \mathcal{J}
$$

with respect to the pre-stable model structures.

Proof All maps in the sets $P, P_{E}$ and $P_{B}$ have cofibrant domains and codomains in the respective categories. Hence this is a consequence of the last lemma and the fact that Quillen equivalences are preserved under left Bousfield localizations [10, Theorem 3.3.20(1b)] applied to the Quillen equivalences in (6-5).

The corollary enables us to give a more explicit characterization of the weak equivalences and the fibrant objects in $\left(\Gamma^{\mathrm{op}} \mathcal{J}-\mathcal{S}\right)_{\text {pre }}$ in terms of ordinary $\Gamma$-spaces and the functor $\gamma^{\prime}: \Gamma^{\mathrm{op}} \mathcal{J}-\mathcal{S} \rightarrow \Gamma^{\mathrm{op}}-\mathcal{S}$ introduced in connection with Corollary 6.16:

Lemma 7.10 A map $f: X \rightarrow Y$ in $\Gamma^{\mathrm{op}} \mathcal{J}-\mathcal{S}$ is a pre-stable equivalence if and only if $\gamma^{\prime}(f)$ is a pre-stable equivalence in $\Gamma^{\mathrm{op}}-\mathcal{S}$. A level fibrant object $X$ is fibrant in $\left(\Gamma^{\mathrm{op}} \mathcal{J}-\mathcal{S}\right)_{\text {pre }}$ if and only if the $\Gamma-$ space $\gamma^{\prime}(X)$ is special.

Proof The strategy of the proof is to compare the zig-zag of Quillen equivalences of level model structures (6-5) with the zig-zag of pre-stable model structures (7-3).

A map $f$ in $\Gamma^{\mathrm{op}} \mathcal{J}-\mathcal{S}$ is a pre-stable equivalence if and only if it represents an isomorphism in $\operatorname{Ho}\left(\left(\Gamma^{\mathrm{op}} \mathcal{J}-\mathcal{S}\right)_{\text {pre }}\right)$. Let $g$ be the image of $f$ under the composed derived 
functor of the zig-zag of level model structures. Then $g$ represents the image of the homotopy class of $f$ under the equivalence $\operatorname{Ho}\left(\left(\Gamma^{\mathrm{op}} \mathcal{J}-\mathcal{S}\right)_{\text {lev }}\right) \rightarrow \operatorname{Ho}\left(\left(\Gamma^{\mathrm{op}}-\mathcal{S}\right)_{\text {lev }} / b \mathcal{J}\right)$ induced by (6-5). Using the equivalence $\operatorname{Ho}\left(\left(\Gamma^{\mathrm{op}} \mathcal{J}-\mathcal{S}\right)_{\text {pre }}\right) \rightarrow \operatorname{Ho}\left(\left(\Gamma^{\mathrm{op}}-\mathcal{S}\right)_{\text {pre }} / b \mathcal{J}\right)$ induced by (7-3), we see that $f$ represents an isomorphism in $\operatorname{Ho}\left(\left(\Gamma^{\mathrm{op}} \mathcal{J}-\mathcal{S}\right)_{\text {pre }}\right)$ if and only if $g$ represents an isomorphism in $\operatorname{Ho}\left(\left(\Gamma^{\mathrm{op}} \mathcal{J}-\mathcal{S}\right)_{\text {pre }} / b \mathcal{J}\right)$. Since the chain of weak equivalences (6-6) induces a chain of level equivalences of $\Gamma$-spaces between $\gamma^{\prime}(f)$ and the underlying map of $\Gamma$-spaces associated with $g$, the claim follows.

An object $X$ is fibrant in $\left(\Gamma^{\mathrm{op}} \mathcal{J}-\mathcal{S}\right)_{\text {pre }}$ if and only if it is $P$-local in the level-model structure. By [10, Proposition 3.1.12] and Lemma 7.6, $X$ is $P$-local if and only if the image $X \times E H \mathcal{J}$ of $X$ under the right adjoint to $\left(\left(\Gamma^{\text {op }} \mathcal{J}-\mathcal{S}\right) / E H \mathcal{J}\right)_{\text {lev }}$ is $P_{E}$-local. Combining [10, Proposition 3.1.12] with [10, Proposition 3.2.2], it follows that an object of $\left(\left(\Gamma^{\mathrm{op}} \mathcal{J}-\mathcal{S}\right) / E H \mathcal{J}\right)_{\text {lev }}$ is $P_{E}-$ local if and only if the left adjoint to $\left(\left(\Gamma^{\mathrm{op}}-\mathcal{S}\right) / b \mathcal{J}\right)_{\text {lev }}$ sends a cofibrant replacement of it to an object that is $P_{B}$-local after level fibrant replacement. Lemma 7.8, [10, Proposition 3.3.16] and the fact that $b \mathcal{J}$ is (very) special show that a fibration over $b \mathcal{J}$ in $\left(\Gamma^{\mathrm{op}}-\mathcal{S}\right)_{\text {pre }}$ is a fibrant object in $\left(\left(\Gamma^{\mathrm{op}}-\mathcal{S}\right) / b \mathcal{J}\right)_{\text {pre }}$ if and only if its source is special. Hence $X$ is fibrant in $\left(\Gamma^{\mathrm{op}} \mathcal{J}-\mathcal{S}\right)_{\text {pre }}$ if and only if it is level fibrant and the underlying $\Gamma$-space of the image of $X$ under the composed derived functor of the zig-zag of Quillen-adjunctions of the level model structures is special. Comparing with $\gamma^{\prime}$ as above proves the claim.

The following result about the relation to commutative $\mathcal{J}$-space monoids is the most difficult step towards Theorem 5.5 and will be proved at the end of this section:

Proposition 7.11 The adjunction $\Phi: \Gamma^{\mathrm{op}} \mathcal{J}-\mathcal{S} \rightleftarrows \mathcal{C S}^{\mathcal{J}}: \Psi$ is a Quillen equivalence with respect to the positive $\mathcal{J}$-model structure on $\mathcal{C S}^{\mathcal{J}}$ and the positive pre-stable model structure on $\Gamma^{\mathrm{op}} \mathcal{J}-\mathcal{S}$.

Together with Corollary 7.9, the proposition implies:

Corollary 7.12 There is a chain of Quillen equivalences

$$
\mathcal{C S}^{\mathcal{J}} \leftrightarrows\left(\Gamma^{\mathrm{op}} \mathcal{J}-\mathcal{S}\right)_{\text {pre }} \leftrightarrows\left(\Gamma^{\mathrm{op}} \mathcal{J}-\mathcal{S} / E H \mathcal{J}\right)_{\text {pre }} \rightleftarrows\left(\Gamma^{\mathrm{op}}-\mathcal{S}\right)_{\text {pre }} / b \mathcal{J}
$$

with respect to the positive $\mathcal{J}$-model structure on $\mathcal{C S}^{\mathcal{J}}$.

\subsection{Stable model structures on $\Gamma^{\text {op }} \mathcal{J}$-spaces}

To get to the stable model structures on $\Gamma^{\mathrm{op}} \mathcal{J}$-spaces and the group completion model structure on $\mathcal{C S}^{\mathcal{J}}$ we are after, we will localize the model categories in Corollary 7.9 one more time. 
For this we consider the maps of finite based sets $d: 2^{+} \rightarrow 1^{+}$and $p_{1}: 2^{+} \rightarrow 1^{+}$ defined by $d(1)=1=d(2)$ and $p_{1}(1)=1, p_{1}(2)=0$. The same arguments as for Lemma 7.3 imply:

Lemma 7.14 The left Bousfield localization of $\left(\Gamma^{\mathrm{op}}-\mathcal{S}\right)_{\text {pre }}$ with respect to the map $d^{*} \vee p_{1}^{*}: \Gamma^{\mathrm{op}}\left(1^{+},-\right) \vee \Gamma^{\mathrm{op}}\left(1^{+},-\right) \rightarrow \Gamma^{\mathrm{op}}\left(2^{+},-\right)$exists.

We call this the stable Q-model structure and write $\left(\Gamma^{\mathrm{op}}-\mathcal{S}\right)_{\mathrm{st}}$ for this model category. It is immediate from the construction that this coincides with the stable Q-model structure on $\Gamma$-spaces considered by Schwede in [27, Theorem 1.5]. This means that a map in $\left(\Gamma^{\mathrm{op}}-\mathcal{S}\right)_{\mathrm{st}}$ is a weak equivalence if the associated map of spectra is a stable equivalence and that an object is fibrant if and only if it is level fibrant and very special. Moreover, the Quillen adjunction $\Gamma^{\mathrm{op}}-\mathcal{S} \rightleftarrows \mathrm{Sp}^{\mathbb{N}}$ exhibits the homotopy category of $\left(\Gamma^{\mathrm{op}}-\mathcal{S}\right)_{\text {st }}$ as the homotopy category of connective spectra. Note that, however, $\left(\Gamma^{\mathrm{op}}-\mathcal{S}\right)_{\mathrm{st}}$ is not a stable model category in the sense that the suspension and loop functor induce inverse equivalences on homotopy categories.

We need again more elaborate sets of maps to localize the various categories of $\Gamma^{\mathrm{op}} \mathcal{J}-$ spaces: as in (7-2), every object $(\boldsymbol{u}, v)$ of $H \mathcal{J}\left(2^{+}\right)$gives rise to a map

$$
\begin{gathered}
\Gamma^{\mathrm{op}} \mathcal{J}\left(\left(1^{+} ;(d)_{*}(\boldsymbol{u}, v)\right),-\right) \amalg \Gamma^{\mathrm{op}} \mathcal{J}\left(\left(1^{+} ;\left(p_{1}\right)_{*}(\boldsymbol{u}, v)\right),-\right) \\
d^{*} \vee p_{1}^{*} \\
\Gamma^{\mathrm{op}} \mathcal{J}\left(\left(2^{+} ; \boldsymbol{u}, v\right),-\right)
\end{gathered}
$$

of free $\Gamma^{\mathrm{op}} \mathcal{J}$-spaces.

Definition 7.15 Let $Q$ be the set of maps in $\Gamma^{\mathrm{op}} \mathcal{J}-\mathcal{S}$ of the form (7-5) where $(\boldsymbol{u}, v)$ is a positive object of $H \mathcal{J}\left(2^{+}\right)$. Let $Q_{E}$ and $Q_{B}$ be defined as the corresponding sets in Definition 7.5.

Applying the functor $\Phi: \Gamma^{\text {op }} \mathcal{J}-\mathcal{S} \rightarrow \mathcal{C} \mathcal{S}^{\mathcal{J}}$ to the set $Q$ provides a set of maps in $\mathcal{C} \mathcal{S}^{\mathcal{J}}$. Up to isomorphism, a map in $\Phi(Q)$ is of the form

$$
\begin{aligned}
& \left(\mathbb{C} F_{\left(\boldsymbol{m}_{1} \sqcup \boldsymbol{n}_{\mathbf{1}}, \boldsymbol{m}_{\mathbf{2}} \sqcup \boldsymbol{n}_{\mathbf{2}}\right)}^{\mathcal{J}}(*)\right) \otimes\left(\mathbb{C} F_{\left(\boldsymbol{n}_{1}, \boldsymbol{n}_{2}\right)}^{\mathcal{J}}(*)\right) \\
& \left(\mathbb{C} F_{\left(\boldsymbol{m}_{\mathbf{1}}, \boldsymbol{m}_{\mathbf{2}}\right)}^{\mathcal{J}}(*)\right) \otimes\left(\mathbb{C} F_{\left(\boldsymbol{n}_{\mathbf{1}}, \boldsymbol{n}_{\mathbf{2}}\right)}^{\mathcal{J}}(*)\right)
\end{aligned}
$$

where $\left(\boldsymbol{m}_{\mathbf{1}}, \boldsymbol{m}_{\mathbf{2}}\right)$ and $\left(\boldsymbol{n}_{\mathbf{1}}, \boldsymbol{n}_{\mathbf{2}}\right)$ run through the positive objects in $\mathcal{J}$.

Lemma 7.16 The left Bousfield localization of the positive $\mathcal{J}$-model structure on $\mathcal{C S}^{\mathcal{J}}$ with respect to $\Phi(Q)$ exists. 
Proof The positive $\mathcal{J}$-model structure on $\mathcal{C S}^{\mathcal{J}}$ is cofibrantly generated and left proper [23, Proposition 4.10]. Since the forgetful functor $\mathcal{C S}^{\mathcal{J}} \rightarrow \mathcal{S}^{\mathcal{J}}$ preserves filtered colimits, it follows from Borceux [6, 5.2.2b and Theorem 5.5.9] that $\mathcal{C} \mathcal{S}^{\mathcal{J}}$ is locally presentable. Hence the existence theorem [4, Theorem 4.7] applies also here.

The resulting model structure $\mathcal{C} \mathcal{S}_{\mathrm{gp}}^{\mathcal{J}}$ will be the group completion model structure of Theorem 5.5.

Counterparts of Lemma 7.6, Lemma 7.7 and Lemma 7.8 for the sets $P \cup Q, P_{E} \cup Q_{E}$ and $P_{B} \cup Q_{B}$ can be proved by the same arguments we used in these lemmas for $P, P_{E}$ and $P_{B}$. This provides stable model structures on $\Gamma^{\mathrm{op}} \mathcal{J}-\mathcal{S}$ and $\Gamma^{\mathrm{op}} \mathcal{J}-\mathcal{S} / E H \mathcal{J}$, and the same arguments as for Corollary 7.9 imply:

Corollary 7.17 There is a chain of Quillen equivalences

$$
\mathcal{C S}_{\mathrm{gp}}^{\mathcal{J}} \leftrightarrows\left(\Gamma^{\mathrm{op}} \mathcal{J}-\mathcal{S} / E H \mathcal{J}\right)_{\mathrm{st}} \rightleftarrows\left(\Gamma^{\mathrm{op}}-\mathcal{S}\right)_{\mathrm{st}} / b \mathcal{J}
$$

with respect to the group completion model structure on $\mathcal{C S}^{\mathcal{J}}$.

Proof of Theorem 5.5 Lemma 7.16 provides the existence of the model structure. It remains to identify the weak equivalences, the fibrant objects and the fibrant replacement. The characterization of the weak equivalences and the fibrant objects in terms of $\gamma$ is completely analogous to Lemma 7.10, this time comparing the zig-zag of Quillen equivalences of pre-stable model structures (7-4) with the zig-zag of stable model structures (7-7). This uses that the stable model structures can be obtained from the pre-stable ones by localizing with respect to $Q_{E}$ and $Q$.

The identification of the weak equivalences and fibrant objects in $\mathcal{C} \mathcal{S}_{\text {gp }}^{\mathcal{J}}$ implies that the fibrant replacement is a group completion in the sense of Definition 5.4.

Remark 7.18 A more direct way to see that fibrant objects in $\mathcal{C S}_{\text {gp }}^{\mathcal{J}}$ are grouplike is that the maps in (7-5) corepresent shear maps $(x, y) \mapsto(x, x+y)$ when applying $\pi_{0}(\operatorname{Map}(-, A))$.

Proof of Theorem 5.10 Corollary 7.17 already gives one possible choice for a zig-zag of Quillen equivalences relating $\mathcal{C S}_{\text {gp }}^{\mathcal{J}}$ and $\left(\Gamma^{\mathrm{op}}-\mathcal{S}\right)_{\mathrm{st}} / b \mathcal{J}$. We used in the proof of Theorem 5.5 that its composed derived functor is stably equivalent to $\gamma$ if we forget the augmentation to $b \mathcal{J}$. As discussed in connection with Corollary 6.30, this is not a stable equivalence over $b \mathcal{J}$. We lift the approach of Lemma 6.31 to $\Gamma^{\mathrm{op}}-\mathcal{S}$ to overcome this: applying the natural map from hocolim $\operatorname{H\mathcal {J}}_{(S)}$ to $\operatorname{colim}_{H \mathcal{J}(S)}$ levelwise defines a level equivalence $\gamma^{\prime}(E H \mathcal{J}) \rightarrow b \mathcal{J}$ which is different from the level equivalence 
$\gamma^{\prime}(E H \mathcal{J}) \rightarrow \gamma^{\prime}(*) \cong b \mathcal{J}$ induced by the map $E H \mathcal{J} \rightarrow *$. Composition with and base change along these maps induces a zig-zag of Quillen equivalences

$$
\left(\Gamma^{\mathrm{op}}-\mathcal{S}\right)_{\mathrm{st}} / b \mathcal{J} \rightleftarrows\left(\Gamma^{\mathrm{op}}-\mathcal{S}\right)_{\mathrm{st}} / \gamma^{\prime}(E H \mathcal{J}) \leftrightarrows\left(\Gamma^{\mathrm{op}}-\mathcal{S}\right)_{\mathrm{st}} / \gamma^{\prime}(*)
$$

and Lemma 6.31 shows that the composite of (7-7) with this zig-zag has a composed derived functor that is stably equivalent to $\gamma$ in $\Gamma^{\mathrm{op}}-\mathcal{S} / b \mathcal{J}$.

\subsection{Proof of Proposition 7.11}

The following lemmas will be used to prove that the adjunction

$$
\Phi:\left(\Gamma^{\mathrm{op}} \mathcal{J}-\mathcal{S}\right)_{\text {pre }} \rightleftarrows \mathcal{C} \mathcal{S}^{\mathcal{J}}: \Psi
$$

is a Quillen equivalence with respect to the positive $\mathcal{J}$-model structure on $\mathcal{C} \mathcal{S}^{\mathcal{J}}$.

Lemma 7.20 The adjunction $\Phi:\left(\Gamma^{\mathrm{op}} \mathcal{J}-\mathcal{S}\right)_{\text {pre }} \rightleftarrows \mathcal{C S}^{\mathcal{J}}: \Psi$ is a Quillen adjunction.

Proof We know from Lemma 6.26 that $(\Phi, \Psi)$ is a Quillen adjunction with respect to the level model structure on $\Gamma^{\mathrm{op}} \mathcal{J}-\mathcal{S}$. So it is enough to show $\Psi$ sends a positive $\mathcal{J}-$ fibration $f: A \rightarrow B$ to a fibration in $\left(\Gamma^{\mathrm{op}} \mathcal{J}-\mathcal{S}\right)_{\text {pre }}$. By Lemma 7.10 and Proposition 3.13, the level fibrant replacements of $\Psi(A)$ and $\Psi(B)$ are fibrant in $\left(\Gamma^{\mathrm{op}} \mathcal{J}-\mathcal{S}\right)_{\text {pre. Since }}$ $\left(\Gamma^{\mathrm{op}} \mathcal{J}-\mathcal{S}\right)_{\text {lev }}$ is right proper, the general criterion [10, Proposition 3.4.7] applies to show that $\Psi(f)$ is a fibration in $\left(\Gamma^{\mathrm{op}} \mathcal{J}-\mathcal{S}\right)_{\text {pre }}$.

Lemma 7.21 A map $f: A \rightarrow B$ in $\mathcal{C S}^{\mathcal{J}}$ is a $\mathcal{J}$-equivalence if and only if $\Psi(f)$ is a pre-stable equivalence in $\Gamma^{\mathrm{op}} \mathcal{J}-\mathcal{S}$.

Proof Lemma 7.10 implies that $\Psi(f)$ is a pre-stable equivalence if and only if $\left(\gamma^{\prime} \Psi\right)(f) \cong \gamma(f)$ is a pre-stable equivalence in $\Gamma^{\mathrm{op}}-\mathcal{S}$. By Proposition 3.13, $\gamma(A)$ and $\gamma(B)$ are special. Hence $\gamma(f)$ is a pre-stable equivalence if and only if it is a weak equivalence when evaluated at $1^{+}$. Because $H \mathcal{J}\left(1^{+}\right) \cong \mathcal{J}$, this holds if and only if $f$ is a $\mathcal{J}$-equivalence.

The right adjoint $\Psi$ preserves coproducts up to weak equivalence:

Lemma 7.22 Let $A$ and $B$ be positive cofibrant commutative $\mathcal{J}$-space monoids, and let $(\Psi(A))^{\text {cof }}$ and $(\Psi(B))^{\text {cof }}$ be cofibrant replacements of $\Psi(A)$ and $\Psi(B)$ in $\left(\Gamma^{\mathrm{op}} \mathcal{J}-\mathcal{S}\right)_{\text {pre }}$. The canonical map

$$
(\Psi(A))^{\mathrm{cof}} \amalg(\Psi(B))^{\mathrm{cof}} \rightarrow \Psi(A \otimes B)
$$

is a pre-stable equivalence. 
Proof By Lemma 7.10, it is enough to show that the map in question induces a pre-stable equivalence after applying $\gamma^{\prime}: \Gamma^{\mathrm{op}} \mathcal{J}-\mathcal{S} \rightarrow \Gamma^{\mathrm{op}}-\mathcal{S}$. Since $\gamma^{\prime}$ is levelwise defined as a homotopy colimit, Corollary 6.8 and the fact that $\left(H \mathcal{J}(S)\left(\mathbf{0}_{S},-\right)\right)_{h H \mathcal{J}(S)}$ is contractible imply that

$$
\gamma^{\prime}\left(\Psi(A)^{\mathrm{cof}}\right) \vee \gamma^{\prime}\left(\Psi(B)^{\mathrm{cof}}\right) \rightarrow \gamma^{\prime}\left(\Psi(A)^{\mathrm{cof}} \amalg \Psi(B)^{\mathrm{cof}}\right)
$$

is a level equivalence. Hence it suffices to show that $\gamma(A) \vee \gamma(B) \rightarrow \gamma(A \otimes B)$ is a pre-stable equivalence in $\Gamma^{\mathrm{op}}-\mathcal{S}$. We claim that the last map factors as

$$
\gamma(A) \vee \gamma(B) \rightarrow \gamma(A) \times \gamma(B) \rightarrow \gamma(A \otimes B),
$$

where $\gamma(A) \times \gamma(B)$ denotes the cartesian product in $\Gamma^{\mathrm{op}}-\mathcal{S}$. (This is not the product in $\Gamma^{\mathrm{op}}-\mathcal{S} / b \mathcal{J}$ !) In each level, the second map in (7-8) is defined as the composite

$$
\begin{gathered}
\left(A_{S}\right)_{h H \mathcal{J}(S)} \times\left(B_{S}\right)_{h H \mathcal{J}(S)} \longrightarrow\left(\left(-\sqcup_{H \mathcal{J}(S)}-\right)^{*}(A \otimes B)_{S}\right)_{h(H \mathcal{J}(S) \times H \mathcal{J}(S))} \\
\downarrow \\
\left((A \otimes B)_{S}\right)_{h H \mathcal{J}(S)}
\end{gathered}
$$

Here $\sqcup_{H \mathcal{J}(S)}$ is the monoidal product in $H \mathcal{J}(S)$, and the map (7-9) is induced by the canonical natural transformation $A_{S} \times B_{S} \rightarrow\left(-\sqcup_{H \mathcal{J}(S)}-\right)^{*}(A \otimes B)_{S}$. We omit the long but straightforward proof that this defines a map of $\Gamma$-spaces. The first map in (7-8) is the canonical map from the coproduct to the product, and the composite is indeed the map we are interested in.

The first map in (7-8) is a pre-stable equivalence because for all pairs of objects in $\operatorname{Ho}\left(\left(\Gamma^{\mathrm{op}}-\mathcal{S}\right)_{\text {pre }}\right)$, the map from the coproduct to the product is an isomorphism. This is a consequence of the equivalence between the homotopy category of special $\Gamma$-spaces and the homotopy category of $E_{\infty}$ spaces [13, Theorem 1.9] and the corresponding statement about the homotopy category of $E_{\infty}$ spaces. The latter follows for example from [23, Theorem 1.2] and [24, Proposition 2.27].

Since $\gamma(A) \times \gamma(B)$ and $\gamma(A \otimes B)$ are special, the second map in (7-8) is a pre-stable equivalence if its evaluation at $1^{+}$is a weak equivalence of spaces. This evaluation is isomorphic to the monoidal structure map $A_{h \mathcal{J}} \times B_{h \mathcal{J}} \rightarrow(A \otimes B)_{h \mathcal{J}}$ of $(-)_{h \mathcal{J}}$. Since $A$ and $B$ are assumed to be cofibrant, their underlying $\mathcal{J}$-spaces are flat in the sense of [23, Section 4.27]. Hence a similar argument as given for the corresponding statement about $\mathcal{I}$-spaces [24, Lemma 2.25] shows that the map is a weak equivalence.

We call a commutative square

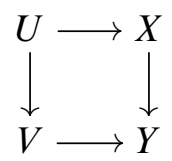


in $\left(\Gamma^{\mathrm{op}} \mathcal{J}-\mathcal{S}\right)_{\text {pre }}$ homotopy cocartesian if for a factorization $U \longmapsto V^{\prime} \stackrel{\sim}{\rightarrow} V$ of $U \rightarrow V$ into a cofibration followed by an acyclic fibration the induced map $V^{\prime} \bigsqcup_{U} X \rightarrow Y$ is a pre-stable equivalence. This is well defined since $\left(\Gamma^{\mathrm{op}} \mathcal{J}-\mathcal{S}\right)_{\text {pre }}$ is left proper and left properness implies the gluing lemma [9, Section II.8]. Homotopy cocartesian squares in $\mathcal{C S}^{\mathcal{J}}$ are defined similarly.

Lemma 7.23 The right adjoint $\Psi$ preserves homotopy cocartesian squares.

Proof Let (7-10) be a homotopy cocartesian test square, let $\mathcal{P}$ be the category $(b \leftarrow a \rightarrow c)$, and let $P: \mathcal{P} \rightarrow \mathcal{C S}^{\mathcal{J}}$ be the diagram $V \leftarrow U \rightarrow X$. In view of Lemma 7.21, we may assume that $\mathcal{P}$ is a cofibrant diagram. This means that all objects are cofibrant and $U \rightarrow V$ and $U \rightarrow X$ are cofibrations. Let $\Psi(P)^{\text {cof }}$ be a cofibrant replacement of $\Psi(P)$ in the category of $\mathcal{P}$-diagrams in $\left(\Gamma^{\text {op }} \mathcal{J}-\mathcal{S}\right)_{\text {pre }}$. Again using Lemma 7.21, it is enough to show that the top map in

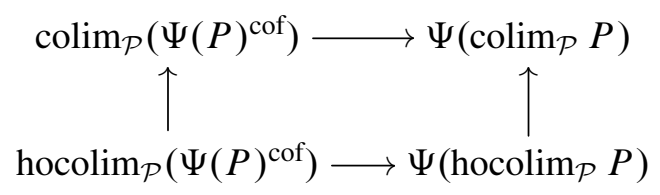

is a weak equivalence. Using the coproducts in the respective categories and the realization functor given by the diagonal, we may also form the Bousfield-Kan homotopy colimits of $P$ and $\Psi(P)^{\text {cof }}$ displayed in the bottom row (compare Definition 2.3). The vertical maps in (7-11) are the canonical maps from the homotopy colimit to the colimit [10, Example 18.3.8] and make the diagram commutative. Since $P$ and $\Psi(P)^{\text {cof }}$ are cofibrant diagrams, an argument similar to [10, Proposition 18.9.4] shows that the vertical maps are weak equivalences.

It remains to show that the bottom map in (7-11) is a weak equivalence. By Lemma 7.22, it is a weak equivalence in every degree of the simplicial replacement used to define the homotopy colimit. Hence Lemma 7.24 shows the claim.

Lemma 7.24 If a map of simplicial objects in $\Gamma^{\mathrm{op}} \mathcal{J}-\mathcal{S}$ is a pre-stable equivalence in every simplicial degree, then its diagonal is a pre-stable equivalence.

Proof The functor $\gamma^{\prime}: \Gamma^{\mathrm{op}} \mathcal{J}-\mathcal{S} \rightarrow \Gamma^{\mathrm{op}}-\mathcal{S}$ and the induced functor on the simplicial objects in these categories commute up to isomorphism with the diagonal. So by Lemma 7.10 it is enough to show that a map $X_{\bullet} \rightarrow Y_{\bullet}$ of simplicial objects in $\Gamma^{\mathrm{op}}-\mathcal{S}$ such that $X_{p} \rightarrow Y_{p}$ is a pre-stable equivalence gives a pre-stable equivalence after taking the diagonal. Viewing $X_{\bullet} \rightarrow Y_{\bullet}$ as a weak equivalence in the Reedy model structure on simplicial objects in $\left(\Gamma^{\mathrm{op}}-\mathcal{S}\right)_{\text {pre }}$, this follows from [10, Theorem 18.6.6] if $X_{\bullet}$ and $Y$. 
are Reedy cofibrant. The general case follows because a Reedy cofibrant replacement $X_{\bullet}^{\prime} \rightarrow X \bullet$ is a Reedy acyclic fibration and hence a degreewise acyclic fibration. These are degreewise level equivalences in $\Gamma^{\mathrm{op}}-\mathcal{S}$ and hence level equivalences after taking the diagonal by the realization lemma for bisimplicial sets.

Lemma 7.25 The adjunction unit $\operatorname{id}_{\Gamma^{\circ} \mathcal{J}_{\mathcal{S}} \mathcal{S}} \rightarrow \Psi \Phi$ is a pre-stable equivalence on the free $\Gamma^{\mathrm{op}} \mathcal{J}$-space $\Gamma^{\mathrm{op}} \mathcal{J}\left(\left(1^{+} ; \boldsymbol{s}, \sigma\right),-\right)$ for every positive object $(\boldsymbol{s}, \sigma)$ of $H \mathcal{J}\left(1^{+}\right)$.

Proof We write $X$ for $\Gamma^{\mathrm{op}} \mathcal{J}\left(\left(1^{+} ; \boldsymbol{s}, \sigma\right),-\right)$ and $\boldsymbol{k}$ for the image of $(\boldsymbol{s}, \sigma)$ under the isomorphism $H \mathcal{J}\left(1^{+}\right) \rightarrow \mathcal{J}$. By Lemma 7.10, it is enough to show that the map

$$
\gamma^{\prime}(X) \rightarrow \gamma^{\prime}(\Psi(\Phi(X))) \cong \gamma(\Phi(X))
$$

is a pre-stable equivalence. The map $\Gamma^{\mathrm{op}}\left(1^{+},-\right) \rightarrow \gamma^{\prime}(X)$ sending $\mathrm{id}_{1+}$ to the 0 simplex in $X\left(1^{+} ;-\right)_{h H \mathcal{J}\left(1^{+}\right)}$specified by $\mathrm{id}_{1^{+}}$and $\mathrm{id}_{\boldsymbol{k}}$ is a level equivalence since the $H \mathcal{J}(T)(T ;-)_{h H \mathcal{J}(T)}$ are contractible.

The definition of $\Phi$ implies that there are isomorphisms

$$
\Phi(X) \cong \mathbb{C} F_{\boldsymbol{k}}^{\mathcal{J}}(*) \cong \coprod_{p \geq 0} \mathcal{J}\left(\boldsymbol{k}^{\sqcup p},-\right) / \Sigma_{p} .
$$

Let $\mathcal{C}_{\boldsymbol{k}}$ be the category whose objects are pairs $([\rho], \boldsymbol{l})$ where $\rho: \boldsymbol{k}^{\sqcup p} \rightarrow \boldsymbol{l}$ is a morphism in $\mathcal{J}$ and $[\rho]$ is its equivalence class in $\mathcal{J}\left(\boldsymbol{k}^{\sqcup p}, \boldsymbol{l}\right) / \Sigma_{p}$. Morphisms in $\mathcal{C}_{\boldsymbol{k}}$ are maps $\varphi: \boldsymbol{l}_{\mathbf{1}} \rightarrow \boldsymbol{l}_{\mathbf{2}}$ such that $\left[\rho_{2}\right]=\left[\varphi \rho_{1}\right]$. Since $\mathbb{C}_{\boldsymbol{k}}^{\mathcal{J}}(*)$ is a $\mathcal{J}$-space with values in discrete simplicial sets, it follows from the definition in the homotopy colimit that $B \mathcal{C}_{\boldsymbol{k}} \cong\left(\mathbb{C F}_{\boldsymbol{k}}^{\mathcal{J}}(*)\right)_{h \mathcal{J}}$. Moreover, $\mathcal{C}_{\boldsymbol{k}}$ is symmetric monoidal with

$$
\left(\left[\rho: \boldsymbol{k}^{\sqcup p} \rightarrow \boldsymbol{l}\right], \boldsymbol{l}\right) \sqcup\left(\left[\rho: \boldsymbol{k}^{\sqcup p^{\prime}} \rightarrow \boldsymbol{l}^{\prime}\right], \boldsymbol{l}^{\prime}\right)=\left(\left[\rho \sqcup \rho^{\prime}: \boldsymbol{k}^{\sqcup p+p^{\prime}} \rightarrow \boldsymbol{l} \sqcup \boldsymbol{l}^{\prime}\right], \boldsymbol{l} \sqcup \boldsymbol{l}^{\prime}\right) .
$$

Inspecting the definitions of Section 3, we see that the last isomorphism extends to an isomorphism of $\Gamma$-spaces $b \mathcal{C}_{\boldsymbol{k}} \cong \gamma\left(\mathbb{C} F_{\boldsymbol{k}}^{\mathcal{J}}(*)\right)$.

Now we let $\Sigma=\operatorname{Iso}(\mathcal{I})$ be the symmetric monoidal category of finite sets and bijections. Sending $\boldsymbol{p}$ to $\left(\left[\operatorname{id}_{\boldsymbol{k}} \sqcup p\right], \boldsymbol{k}^{\sqcup p}\right)$ defines a symmetric monoidal functor $\Sigma \rightarrow \mathcal{C}_{\boldsymbol{k}}$. It induces a map of special $\Gamma$-spaces $b \Sigma \rightarrow \Gamma\left(\mathbb{C F}_{\boldsymbol{k}}^{\mathcal{J}}(*)\right)$ which is a level equivalence because

$$
b \Sigma\left(1^{+}\right) \rightarrow \gamma\left(\mathbb{C} F_{\boldsymbol{k}}^{\mathcal{J}}(*)\right)\left(1^{+}\right) \cong\left(\mathbb{C} F_{\boldsymbol{k}}^{\mathcal{J}}(*)\right)_{h \mathcal{J}} \simeq \coprod_{p \geq 0} B \Sigma_{p}
$$

is a weak equivalence. The last weak equivalence uses that $\boldsymbol{k}$ is positive and follows from a similar argument as in the case of $\mathcal{I}$-spaces [24, Example 3.7].

Segal's proof of the Barratt-Priddy-Quillen theorem [29, Proposition 3.5] implies that the map $\Gamma^{\mathrm{op}}\left(1^{+},-\right) \rightarrow b \Sigma$ sending $\mathrm{id}_{1^{+}}$to the component of $\mathrm{id}_{1}$ in $(b \Sigma)\left(1^{+}\right)$induces 
an isomorphism in the homotopy category of special $\Gamma$-spaces. So $\Gamma^{\mathrm{op}}\left(1^{+},-\right) \rightarrow b \Sigma$ is a pre-stable equivalence. Because the component of $\mathrm{id}_{\boldsymbol{k}}$ is in the image of

$$
\pi_{0}\left(\gamma^{\prime}(X)\left(1^{+}\right)\right) \rightarrow \pi_{0}\left(\gamma\left(\mathbb{C} F_{\boldsymbol{k}}^{\mathcal{J}}(*)\right)\left(1^{+}\right)\right),
$$

the above level equivalences shows that $\gamma^{\prime}(X) \rightarrow \gamma(\Phi(X))$ is a pre-stable equivalence.

Lemma 7.26 The adjunction unit $\operatorname{id}_{\Gamma^{\circ} \mathcal{J}_{\mathcal{S}} \mathcal{S}} \rightarrow \Psi \Phi$ is a pre-stable equivalence on the $\Gamma^{\mathrm{op}} \mathcal{J}$-space $\Gamma^{\mathrm{op}} \mathcal{J}((S ; \boldsymbol{s}, \sigma),-) \otimes K$ for every positive object $(\boldsymbol{s}, \sigma)$ of $H \mathcal{J}(S)$ and every finite simplicial set $K$.

Proof We write $X$ for the $\Gamma^{\mathrm{op}} \mathcal{J}$-space $\Gamma^{\mathrm{op}} \mathcal{J}((S ; \boldsymbol{s}, \sigma),-)$ and show first that the map $X \rightarrow \Psi \Phi(X)$ is a pre-stable equivalence. Setting $X_{i}=\Gamma^{\text {op }} \mathcal{J}\left(\left(S ;\left(p_{i}\right)_{*}(s, \sigma)\right),-\right)$, the maps $(S ; \boldsymbol{s}, \sigma) \rightarrow\left(1^{+} ;\left(p_{i}\right)_{*}(\boldsymbol{s}, \sigma)\right)$ induce a commutative diagram

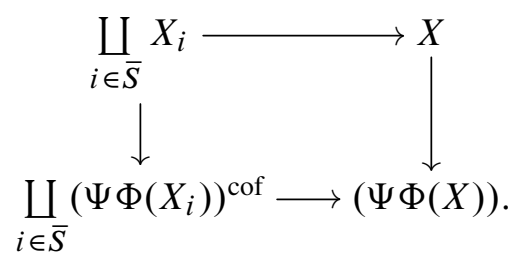

The top horizontal map is a pre-stable equivalence by the definition of the set $P$ used to construct the localization. Because $\bigsqcup_{i \in \bar{S}} \Phi\left(X_{i}\right) \rightarrow \Phi(X)$ is an isomorphism, Lemma 7.22 implies that the bottom horizontal map is a pre-stable equivalence. By Lemma 7.25, the left map is a coproduct of pre-stable equivalences between cofibrant objects. It follows that $X \rightarrow(\Psi \Phi(X))$ is a pre-stable equivalence.

To show that $X \otimes K \rightarrow(\Psi \Phi(X \otimes K))$ is a pre-stable equivalence we first note that for a $\Gamma^{\text {op }} \mathcal{J}$-space $Y$ there is an isomorphism

$$
Y \rightarrow K=Y \wedge K_{+} \cong\left|[m] \mapsto \coprod_{K_{m}} Y\right| .
$$

Choosing a cofibrant replacement

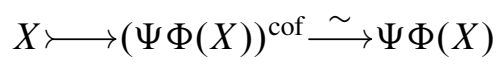

of $\Psi \Phi(X)$, the universal property of the coproduct induces a map

$$
\left|[m] \mapsto \coprod_{K_{m}}(\Psi \Phi(X))^{\operatorname{cof}}\right| \longrightarrow\left|[m] \mapsto\left(\Psi \Phi\left(\coprod_{K_{m}} X\right)\right)^{\operatorname{cof}}\right| .
$$


Under the isomorphism (7-12), this becomes $(\Psi \Phi(X))^{\text {cof }} \otimes K \rightarrow(\Psi \Phi(X \otimes K))$. Applying Lemma 7.22 and Lemma 7.24 to (7-13) shows that the last map is a pre-stable equivalence. Precomposing it with $\left(X \rightarrow(\Psi \Phi(X))^{\text {cof }}\right) \otimes K$ gives the adjunction unit on $X \otimes K$. Since the pre-stable model structure is simplicial and we already showed that $X \rightarrow \Psi \Phi(X)$ is a pre-stable equivalence, the claim follows by the 2-out-of-3 property for pre-stable equivalences.

Proof of Proposition 7.11 We use the criterion of [11, Corollary 1.3.16(c)] to see that $(\Phi, \Psi)$ is a Quillen equivalence. In view of Lemma 7.20 and Lemma 7.21, it remains to show that the adjunction unit $X \rightarrow \Psi \Phi(X)$ is a pre-stable equivalence for all cofibrant objects $X$ in $\left(\Gamma^{\mathrm{op}} \mathcal{J}-\mathcal{S}\right)_{\text {pre }}$.

Lemma 7.26 and Corollary 6.10 imply that the adjunction unit is a pre-stable equivalence on the domains and codomains of the generating cofibrations. The adjunction unit is also a pre-stable equivalence on the initial object in $\Gamma^{\mathrm{op}} \mathcal{J}-\mathcal{S}$ because $\Phi$ maps it to the initial commutative $\mathcal{J}$-space monoid $F_{\mathbf{0}}^{\mathcal{J}}(*)$ and $\left(F_{\mathbf{0}}^{\mathcal{J}}(*)\right)_{h \mathcal{J}}$ is contractible.

Now let $V \longleftarrow U \rightarrow X$ be a diagram of cofibrant objects in $\left(\Gamma^{\text {op }} \mathcal{J}-\mathcal{S}\right)_{\text {pre }}$ such that the adjunction unit is a pre-stable equivalence on $U, V$ and $X$. The fact that the left Quillen functor $\Phi$ preserves homotopy cocartesian squares of cofibrant objects, Lemma 7.23, and the gluing lemma in $\left(\Gamma^{\mathrm{op}} \mathcal{J}-\mathcal{S}\right)_{\text {pre }}$ imply that the adjunction unit on the pushout of the diagram is a pre-stable equivalence.

Next let $\cdots \rightarrow X_{i} \rightarrow X_{i+1} \rightarrow \cdots$ be a sequence of cofibrations between cofibrant objects in $\left(\Gamma^{\mathrm{op}} \mathcal{J}-\mathcal{S}\right)_{\text {pre }}$ such that the unit is a pre-stable equivalence on every $X_{i}$. We claim that it is a pre-stable equivalence on $X=\operatorname{colim} X_{i}$. The forgetful functor $\mathcal{C S}^{\mathcal{J}} \rightarrow \mathcal{S}^{\mathcal{J}}$ preserves filtered colimits. Hence the composite $\Psi \Phi$ commutes with sequential colimits, and it is enough to show that the map colim $X_{i} \rightarrow \operatorname{colim} \Psi\left(\Phi\left(X_{i}\right)\right)$ is a pre-stable equivalence. Since the maps between the $X_{i}$ are cofibrations, the map hocolim $X_{i} \rightarrow \operatorname{colim} X_{i}$ is a level equivalence. Combining [23, Proposition 7.1(v)] and [23, Proposition 12.7] with the fact that filtered colimits commute with products implies that hocolim $\Psi\left(\Phi\left(X_{i}\right)\right) \rightarrow \operatorname{colim} \Psi\left(\Phi\left(X_{i}\right)\right)$ is a level equivalence. Together with the homotopy invariance of the homotopy colimit this shows that $X \rightarrow \Psi(\Phi(X))$ is a pre-stable equivalence.

Every cofibrant object $X$ in $\left(\Gamma^{\mathrm{op}} \mathcal{J}-\mathcal{S}\right)_{\text {pre }}$ is the retract of the colimit of a sequence of cofibrations starting at the initial object in which all maps are cobase changes of the generating cofibrations. So the above implies that the adjunction unit is a pre-stable equivalence on all cofibrant objects. 


\section{References}

[1] M Ando, A J Blumberg, D Gepner, Parametrized spectra, multiplicative Thom spectra, and the twisted Umkehr map, preprint (2015) arXiv:1112.2203

[2] M Ando, A J Blumberg, D Gepner, M J Hopkins, C Rezk, An $\infty$-categorical approach to $R$-line bundles, $R$-module Thom spectra, and twisted $R$-homology, J. Topol. 7 (2014) 869-893 MR3252967

[3] M Ando, A J Blumberg, D Gepner, M J Hopkins, C Rezk, Units of ring spectra, orientations and Thom spectra via rigid infinite loop space theory, J. Topol. 7 (2014) 1077-1117 MR3286898

[4] C Barwick, On left and right model categories and left and right Bousfield localizations, Homology, Homotopy Appl. 12 (2010) 245-320 MR2771591

[5] J M Boardman, R M Vogt, Homotopy invariant algebraic structures on topological spaces, Lecture Notes in Mathematics 347, Springer, Berlin (1973) MR0420609

[6] F Borceux, Handbook of categorical algebra, 2, Encyclopedia of Mathematics and its Applications 51, Cambridge Univ. Press (1994) MR1313497

[7] A K Bousfield, E M Friedlander, Homotopy theory of $\Gamma$-spaces, spectra, and bisimplicial sets, from: "Geometric applications of homotopy theory", Lecture Notes in Math. 658, Springer, Berlin (1978) 80-130 MR513569

[8] B I Dundas, T G Goodwillie, R McCarthy, The local structure of algebraic $K$-theory, Algebra and Applications 18, Springer, London (2013) MR3013261

[9] P G Goerss, J F Jardine, Simplicial homotopy theory, Progress in Mathematics 174, Birkhäuser, Basel (1999) MR1711612

[10] PS Hirschhorn, Model categories and their localizations, Mathematical Surveys and Monographs 99, Amer. Math. Soc. (2003) MR1944041

[11] M Hovey, Model categories, Mathematical Surveys and Monographs 63, Amer. Math. Soc. (1999) MR1650134

[12] J A Lind, Diagram spaces, diagram spectra and spectra of units, Algebr. Geom. Topol. 13 (2013) 1857-1935 MR3073903

[13] MA Mandell, An inverse K-theory functor, Doc. Math. 15 (2010) 765-791 MR2735988

[14] M A Mandell, J P May, S Schwede, B Shipley, Model categories of diagram spectra, Proc. London Math. Soc. 82 (2001) 441-512 MR1806878

[15] J P May, The geometry of iterated loop spaces, Lecture Notes in Mathematics 271, Springer, Berlin (1972) MR0420610

[16] J P May, $E_{\infty}$ ring spaces and $E_{\infty}$ ring spectra, Lecture Notes in Mathematics 577, Springer, Berlin (1977) MR0494077 
[17] J Rognes, Topological logarithmic structures, from: "New topological contexts for Galois theory and algebraic geometry", (A Baker, B Richter, editors), Geom. Topol. Monogr. 16, Coventry (2009) 401-544 MR2544395

[18] J Rognes, Algebraic K-theory of strict ring spectra, from: "Proceedings of the International Congress of Mathematicians, Vol. II”, Kyung Moon Sa, Seoul (2014) 1259-1283

[19] J Rognes, S Sagave, C Schlichtkrull, Localization sequences for logarithmic topological Hochschild homology, Math. Ann. 363 (2015) 1349-1398 MR3412362

[20] J Rognes, S Sagave, C Schlichtkrull, Logarithmic topological Hochschild homology of topological K-theory spectra, preprint (2015) arXiv:1410.2170

[21] S Sagave, Logarithmic structures on topological $K$-theory spectra, Geom. Topol. 18 (2014) 447-490 MR3159166

[22] S Sagave, C Schlichtkrull, Graded units of ring spectra and graded Thom spectra In preparation

[23] S Sagave, C Schlichtkrull, Diagram spaces and symmetric spectra, Adv. Math. 231 (2012) 2116-2193 MR2964635

[24] S Sagave, C Schlichtkrull, Group completion and units in $\mathscr{I}$-spaces, Algebr. Geom. Topol. 13 (2013) 625-686 MR3044590

[25] S Sagave, C Schlichtkrull, Virtual vector bundles and graded Thom spectra, preprint (2014) arXiv:1410.4492

[26] C Schlichtkrull, Units of ring spectra and their traces in algebraic $K$-theory, Geom. Topol. 8 (2004) 645-673 MR2057776

[27] S Schwede, Stable homotopical algebra and $\Gamma$-spaces, Math. Proc. Cambridge Philos. Soc. 126 (1999) 329-356 MR1670249

[28] S Schwede, Symmetric spectra, Book project in progress (2007) Available at http:// www.math. uni-bonn.de/people/schwede/SymSpec.pdf

[29] G Segal, Categories and cohomology theories, Topology 13 (1974) 293-312 MR0353298

[30] N Shimada, K Shimakawa, Delooping symmetric monoidal categories, Hiroshima Math. J. 9 (1979) 627-645 MR549667

Institute for Mathematics, Astrophysics and Particle Physics, Radboud University Nijmegen PO Box 9010, 6500 GL Nijmegen, The Netherlands

s.sagave@math.ru.nl

http://www.math.ru.nl/ sagave/

Received: 26 June 2015 Revised: 11 July 2015 
